\title{
Adaptation of iCLIP to plants determines the binding landscape of the clock- regulated RNA-binding protein AtGRP7
}

Katja Meyer ${ }^{1 \dagger}$, Tino Köster ${ }^{1 \dagger}$, Christine Nolte ${ }^{1}$, Claus Weinholdt ${ }^{2}$, Martin Lewinski ${ }^{1}$, Ivo Grosse ${ }^{2,3}$ and Dorothee Staiger ${ }^{1 *}$

\begin{abstract}
Background: Functions for RNA-binding proteins in orchestrating plant development and environmental responses are well established. However, the lack of a genome-wide view of their in vivo binding targets and binding landscapes represents a gap in understanding the mode of action of plant RNA-binding proteins. Here, we adapt individual nucleotide resolution crosslinking and immunoprecipitation (iCLIP) genome-wide to determine the binding repertoire of the circadian clock-regulated Arabidopsis thaliana glycine-rich RNA-binding protein AtGRP7.

Results: iCLIP identifies 858 transcripts with significantly enriched crosslink sites in plants expressing AtGRP7-GFP that are absent in plants expressing an RNA-binding-dead AtGRP7 variant or GFP alone. To independently validate the targets, we performed RNA immunoprecipitation (RIP)-sequencing of AtGRP7-GFP plants subjected to formaldehyde fixation. Of the iCLIP targets, 452 were also identified by RIP-seq and represent a set of high-confidence binders. AtGRP7 can bind to all transcript regions, with a preference for $3^{\prime}$ untranslated regions. In the vicinity of crosslink sites, U/C-rich motifs are overrepresented. Crossreferencing the targets against transcriptome changes in AtGRP7 loss-of-function mutants or AtGRP7-overexpressing plants reveals a predominantly negative effect of AtGRP7 on its targets. In particular, elevated AtGRP7 levels lead to damping of circadian oscillations of transcripts, including DORMANCY/AUXIN ASSOCIATED FAMILY PROTEIN2 and CCR-LIKE. Furthermore, several targets show changes in alternative splicing or polyadenylation in response to altered AtGRP7 levels.
\end{abstract}

Conclusions: We have established iCLIP for plants to identify target transcripts of the RNA-binding protein AtGRP7. This paves the way to investigate the dynamics of posttranscriptional networks in response to exogenous and endogenous cues.

Keywords: Circadian rhythm, Individual nucleotide resolution crosslinking and immunoprecipitation (iCLIP), RNA immunoprecipitation (RIP), RNA-binding protein

\section{Background}

RNA-binding proteins (RBPs) regulate RNA processing steps from synthesis to decay, including pre-mRNA splicing, transport, $3^{\prime}$ end formation, translation, and degradation. This regulation at the RNA level represents an important checkpoint to extensively modulate gene expression once transcription has been initiated. Arabidopsis thaliana harbors 197 proteins with an RNA recognition motif (RRM), the most frequent type of RNA-binding

\footnotetext{
* Correspondence: dorothee.staiger@uni-bielefeld.de

${ }^{\dagger}$ Equal contributors

${ }^{1}$ RNA Biology and Molecular Physiology, Faculty of Biology, Bielefeld

University, Bielefeld, Germany

Full list of author information is available at the end of the article
}

domain [1]. The complete binding repertoire of any of these RBPs is virtually unknown.

To date, global mapping of in vivo RNA-protein interactions is performed by immunopurification of RNA-binding proteins using antibodies against the native protein or an epitope, and cataloguing the associated RNAs by RNA-seq. In higher plants, RBPs were immunoprecipitated from lysates of purified maize chloroplasts under native conditions and RNAs were identified by microarrays [2]. To preserve the physiological RNA-protein interactions, RNA and bound proteins are often crosslinked in vivo. In conventional RNA immunoprecipitation (RIP) techniques, formaldehyde is used for crosslinking. RIP and subsequent identification of bound transcripts by reverse transcription (RT)-PCR has been used 
to confirm candidate in vivo targets of plant RBPs [3, 4]. In a first RIP-seq analysis in Arabidopsis, more than 4000 targets of the serine/arginine rich (SR)-like protein SR45 were identified by RNA immunoprecipitation, followed by highthroughput sequencing [5].

While RIP is useful to identify in vivo target transcripts, it does not provide immediate information about the binding motifs on the RNAs. To overcome this drawback, more recently developed crosslinking and immunoprecipitation (CLIP) techniques rely on UV-induced covalent bonds between RBPs and their target RNAs, providing information on the site of interaction [6]. For CLIP, adapters are attached to both the $5^{\prime}$ and $3^{\prime}$ ends of the RNAs co-precipitating with the protein of interest. Thus, CLIP can only identify sequences with read through of the RT beyond the crosslink site. However, up to $80 \%$ of the cDNAs terminate at the crosslinked nucleotide [7-9]. This property has been used to increase the resolution in individual nucleotide resolution crosslinking and immunoprecipitation (iCLIP) [10]. A linker is ligated to the $3^{\prime}$ end of the RNAs, serving as a docking platform for a two-part cleavable RT primer. Circularization of the cDNAs and relinearization places part of the adapter to the $5^{\prime}$ end so that truncated cDNAs are captured for preparation of the RNA-seq libraries.

CLIP techniques have initially been developed for cells cultured in monolayers or mammalian tissue $[6,10]$. Thus, it was not clear whether intact plants with UVabsorbing pigments would allow efficient crosslinking, and whether the UV light treatment could elicit UV stress responses in plants that might compromise physiological RNA-protein interactions.

We chose to establish iCLIP for Arabidopsis, using Arabidopsis thaliana glycine-rich RNA-binding protein 7 (AtGRP7) as a paradigm. AtGRP7 is controlled by the circadian clock, an endogenous timekeeper that prepares organisms for the periodic changes of day and night [11]. AtGRP7 consists of a single RRM and a namesake glycine-rich stretch. The AtGRP7 transcript oscillates with a peak in the evening, and the oscillations persist in continuous light [12, 13]. Ectopic over-expression of AtGRP7 (AtGRP7-ox) leads to damping of the endogenous AtGRP7 transcript oscillations: Binding of AtGRP7 to its own pre-mRNA causes a shift to an alternative splice form retaining part of the intron with a premature termination codon (PTC) that is degraded via nonsensemediated decay (NMD) [14, 15]. Elevated levels of AtGRP7 also negatively regulate the paralog AtGRP8 through alternative splicing and NMD. Furthermore, AtGRP7 regulates alternative splicing of a suite of downstream targets [16]. Additionally, AtGRP7 functions as an RNA chaperone [17]. Mutation of the conserved Arg49 in the RNA-binding domain $\left(\mathrm{R}^{49} \mathrm{Q}\right)$ abolishes in vivo RNA binding and function [18, 19]. AtGRP7 is involved in a suite of physiological processes, including circadian timekeeping, cold responses, phytohormone responses, and flowering time control [20-22]. To comprehensively understand how AtGRP7 exerts its diverse functions, determination of its target transcripts and binding landscape at a genome-wide scale is of central importance.

Here, we determined AtGRP7 targets by iCLIP and a parallel RIP-seq analysis for independent validation. In plants expressing an AtGRP7-GREEN FLUORESCENT PROTEIN (GFP) fusion we identified significant crosslink sites in 858 target transcripts that were not detected in plants expressing the RNA-binding dead variant AtGRP7 $\mathrm{R}^{49} \mathrm{Q}-\mathrm{GFP}$, or GFP alone. Of these targets, 452 were also identified by RIP-seq following formaldehyde crosslinking, defining a set of high-confidence binders. In the vicinity of the crosslink sites, UC rich motifs were enriched. To investigate whether the identified in vivo targets are regulated by AtGRP7 at the mRNA level, we performed total RNA-seq of AtGRP7 loss-of-function and overexpressing plants. Direct binding targets appear to be predominantly negatively regulated by AtGRP7. In particular, circadian transcript oscillations are damped in AtGRP7-overexpressing plants.

\section{Results}

To identify in vivo binding targets of the circadian clock-regulated RBP AtGRP7 by iCLIP at a genome-wide scale, GFP-tagged AtGRP7 was expressed under control of its own promoter including the $5^{\prime}$ UTR, 3' UTR, and intron (AtGRP7::AtGRP7-GFP) in the grp7-1 loss-offunction mutant [21]. This construct recapitulates the endogenous expression pattern, thus reducing binding to non-physiological targets due to aberrantly high or ectopic AtGRP7 expression, and it enables efficient immunoprecipitation using GFP Trap beads with the high affinity single chain antibodies [23].

\section{Conditions for UV light crosslinking of RNA-protein complexes in Arabidopsis plants}

To covalently crosslink RNA binding targets to the AtGRP7-GFP fusion protein in vivo, we adapted UV crosslinking (XL) established for mammalian cells, yeast, or Caenorhabditis elegans to Arabidopsis plants [10, 24]. Sixteen-day-old AtGRP7:AtGRP7-GFP grp7-1 plants were subjected to irradiation with UV-C light $(254 \mathrm{~nm})$ at a dose of $500 \mathrm{~mJ} / \mathrm{cm}^{2}$. To test how this UV-C treatment affects the physiological state of the plants, we first monitored the METACASPASE 8 (MC8) transcript that is known to be upregulated in response to UV stress [25, 26]. We detected a significant increase in $M C 8$ only after $1 \mathrm{~h}$, indicating that UV stress-induced changes in gene expressions are negligible within the few minutes between treatment and harvest (Additional file 1: Figure S1a, b). As UV-C is also known to trigger the HY5-mediated UV-B signaling 


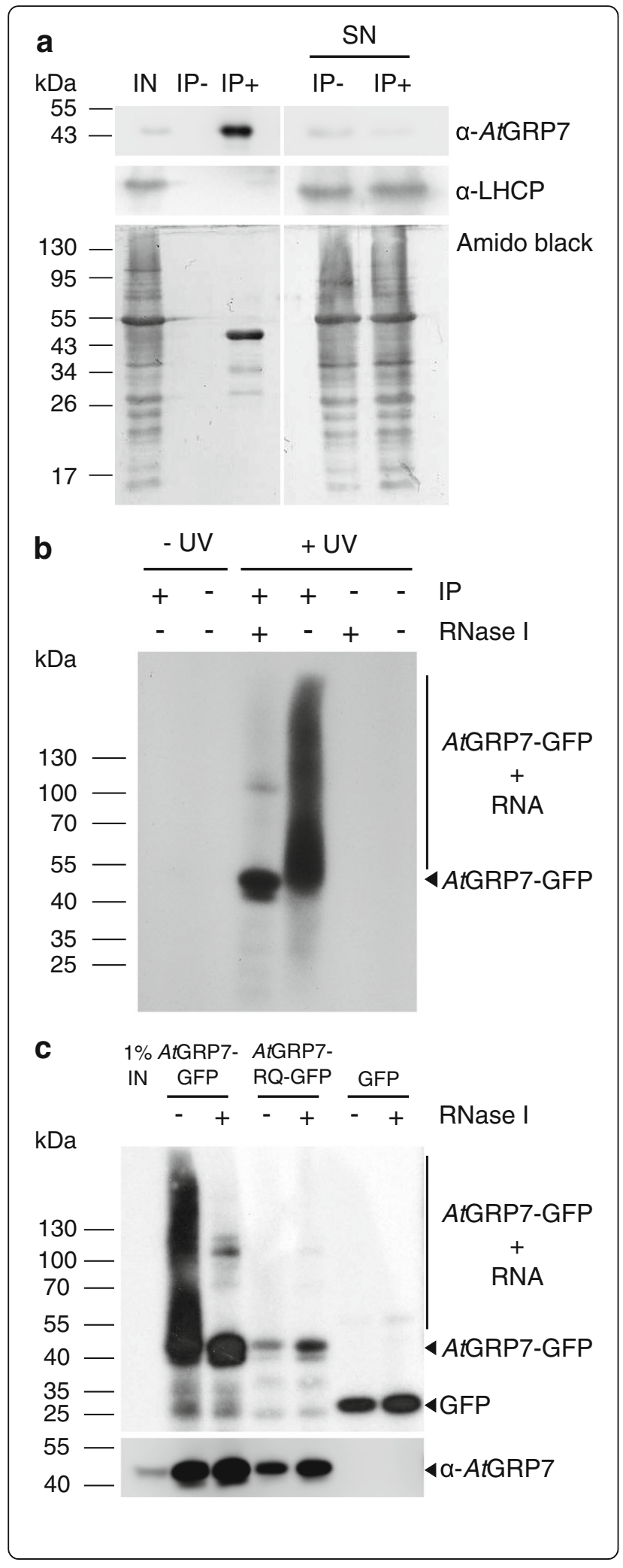

Fig. 1 Immunoprecipitation of AtGRP7 protein-RNA complexes from UV crosslinked AtGRP7:AtGRP7-GFP grp7-1 plants. RNA-protein interactions were stabilized by UV irradiation of 16-day-old plants with UV light $(254 \mathrm{~nm})$ at $500 \mathrm{~mJ} / \mathrm{cm}^{2}$. Lysates were subjected to immunoprecipitation with GFP Trap beads $(I P+)$ and mock precipitation with RFP Trap beads $(I P-)$. a Aliquots of the lysate (input, $I N), I P+, I P-$ and the supernatant (SM) of the precipitations were analyzed by immunoblotting with the a-AtGRP7 antibody. The a-LHCP antibody served as control. For comparison, the membrane was stained with amidoblack. Positions of the molecular weight markers are indicated. $\mathbf{b}$ Autoradiogram of RNA-protein complexes from AtGRP7:AtGRP7-GFP grp7-1 plants after UV XL and without UV XL and after precipitation $(I P+)$ or mock precipitation $(I P-)$. Treatment of the precipitate with RNase I ( $R$ Nase) indicates the size of the precipitated protein. c Autoradiogram of RNA-protein complexes of UV crosslinked AtGRP7:AtGRP7-GFP grp7-1 plants, AtGRP7:AtGRP7 R ${ }^{49} \mathrm{Q}-$ GFP, and AtGRP7::GFP-only plants. Immunoblot against AtGRP7 identifies the precipitated protein (bottom). Marker positions and the location of the AtGRP7-GFP RNA adducts are indicated

pathway, we monitored the level of $H Y 5$ HOMOLOG $(H Y H)$, a marker for UV-B photomorphogenesis [27]. $H Y H$ also showed a significant increase only $60 \mathrm{~min}$ after irradiation. The level of the AtGRP7-GFP fusion protein itself did not change upon UV treatment, excluding false positive results due to a UV effect on AtGRP7 (Additional file 1: Figure S1c).

As UV-C light also leads to programmed cell death in Arabidopsis [28], we checked whether the treatment with 254-nm UV light causes long-term damage by visible inspection of the plants $2,5,6$, and 8 days after irradiation (Additional file 1: Figure S1d). After 5 days, irradiated leaves showed complete bleaching, but at the same time new, green leaves emerged. Taken together, these data showed that UV light at the dose chosen indeed reaches the interior of the leaves but does not noticeably change the physiological state of the plants in the timeframe before harvest, suggesting that we would obtain a valid snapshot of the AtGRP7 RNome at the time of irradiation under these conditions.

Next, we confirmed an efficient recovery of the AtGRP7GFP fusion protein from the lysate upon precipitation with GFP Trap beads (IP+) but not upon mock precipitation with RED FLUORESCENT PROTEIN (RFP) Trap beads (IP-) by immunoblotting (Fig. 1a). The light harvesting chlorophyll binding protein LHCP was not depleted from the supernatant.

To optimize the yield of the RNA-protein complexes, we adapted the lysate preparation for iCLIP on the basis of the protocol we have developed for RIP following formaldehyde fixation $[4,29]$. In particular, higher concentrations of ionic detergent (1\% SDS) than used for mammalian cells $(0.1 \%$ SDS) [10] led to more efficient protein extraction in the lysate. The formation of covalent AtGRP7-GFP-RNA adducts upon UV crosslinking was monitored by radiolabeling of the RNA. Upon SDS-PAGE, blotting and autoradiography, 
complexes were detected in crosslinked AtGRP7:AtGRP7GFP grp7-1 plants but not in non-crosslinked plants (Fig. 1b). No complexes were precipitated by RFP Trap beads (IP-) irrespective of UV irradiation. This suggested that the co-precipitating RNAs were mostly targets of the RBP and not only RNAs interacting nonspecifically with the beads. RNase I treatment eliminated most of the crosslinked RNA. As additional controls we used plants expressing the AtGRP7::AtGRP7 $R^{49} Q-G F P$ variant with reduced RNA-binding activity due to mutation of a conserved arginine residue in the RRM, or the GFP moiety alone, under control of the AtGRP7 promoter. Only little RNA-protein complexes were precipitated in these controls (Fig. 1c). Probing of the membrane with the AtGRP7 antibody confirmed the identity of the precipitated proteins (Fig. 1c).

\section{iCLIP of AtGRP7}

To comprehensively identify binding substrates of AtGRP7, libraries were prepared from the RNA-protein complexes for sequencing on the Illumina platform. The procedure is schematically shown in Additional file 1: Figure S2a. AtGRP7::AtGRP7-GFP grp7-1 plants were grown in $12 \mathrm{~h}$ light $-12 \mathrm{~h}$ dark cycles, and transferred to continuous light. UV crosslinking was performed after $36 \mathrm{~h}$, at subjective dusk (LL36), the time of AtGRP7 peak expression. After immunoprecipitation of the RNA-protein complexes from the lysate, the membrane region corresponding to the smear of covalently linked AtGRP7-GFP-RNA complexes was excised (Additional file 1: Figure S2b). RNA was extracted and libraries were prepared as described in "Methods". In parallel, negative control libraries were prepared from corresponding regions of lanes containing RNA-protein complexes from GFP-only plants and AtGRP7:AtGRP7 $R^{49}$ Q-GFP plants (representative samples shown in Additional file 1: Figure S2b). The read statistics of the individual replicates are shown in Additional file 2: Table S1.

Raw iCLIP reads were subjected to 3 ' adapter trimming, quality filtering, and de-multiplexing. PCR duplicates were removed and the barcodes were trimmed. The resulting reads were mapped to the $A$. thaliana TAIR10 reference genome using the additional transcript annotation file atRTD.gff from the reference transcriptome atRTD [30]. Only reads mapping uniquely were kept. Putative crosslink sites were determined separately for each transcript region essentially as described [10]. Only the position one nucleotide upstream of the read start was considered, which represents the XL site. Based on the amount of reads at this site, a false discovery rate (FDR) determined whether an XL site was significantly different from a randomly generated background (see "Methods" for details).

In total, 96,307 significant crosslink sites were identified in the five AtGRP7:AtGRP7-GFP grp7-1 replicates. To extract the most robust XL sites, they were required to map to the same position in independent biological replicates. We found 11,021 enriched XL sites in 865 transcripts for AtGRP7-GFP located at the same position in at least four of the five biological replicates. In the GFP-only plants, 162 significant XL sites were found (0.13\% of the XL sites in AtGRP7::AtGRP7-GFP grp7-1 plants). These occurred rarely at the same positions in the five independent biological replicates, suggesting that they represent background interactions (Additional file 1: Figure S3). Ten crosslink sites in six transcripts were present at the same position in at least four of the five replicates, and these transcripts were also among the 865 candidate AtGRP7 targets. In the AtGRP7::AtGRP7 $R^{49}$ Q-GFP plants, 349 significant XL sites were identified. Only three XL sites were identified at the same position in two replicates and two of those appeared also in the GFP-only plants (Additional file 2: Table S2). All transcripts with significant XL sites in either GFP-only plants, AtGRP7 $\mathrm{R}^{49} \mathrm{Q}$-GFP plants, or both were not considered further, leaving 858 candidate AtGRP7 target transcripts (Additional file 2: Table S2).

The localization of the significant XL sites within these transcripts was determined. After normalizing the number of XL sites in each region relative to the overall length of the transcript region in the genome, most $\mathrm{XL}$ sites mapped to the 3' UTR and the 5' UTR (Fig. 2a). The lowest number was observed in the introns: 258 transcripts contained XL sites only in the 3' UTR, 136 transcripts only in the exons (coding region), 78 transcripts only in the $5^{\prime}$ UTR, and 70 transcripts only in the introns (Fig. 2b). Many transcripts contained more than one significant XL site, both in the same region and in different regions of the transcripts; e.g., 118 transcripts contained XL sites in both the 3' UTR and the exons (Fig. 2b). The binding profiles of selected AtGRP7 targets are shown in Fig. 3 and Additional file 1: Figure S4.

The circadian clock-regulated DORMANCY/AUXIN ASSOCIATED FAMILY PROTEIN (DRM2; At2g33830) and CCR-LIKE (CCL; At3g26740) transcripts showed XL sites in the 5' UTR, exon 1, and the 3' UTR, and another clock-regulated transcript, ARABIDOPSIS THALIANA ALUMINIUM-INDUCED-LIKE PROTEIN 1 (AILP1; At5g19140), showed XL sites in both the 5' UTR and 3' UTR (Fig. 3a-c). ORTHOLOG OF SUGAR BEET HS1 PRO-1 2 (ATHSPRO2; At2g40000), which is involved in basal resistance against Pseudomonas syringae, showed XL sites in the exon (Fig. 3d). FERREDOXIN NAD(P)H OXIDOREDUCTASE 2 (FNR2; At1g20020) displayed XL sites in the $5^{\prime}$ UTR, exons 1,4 , and 5, as well as the $3^{\prime}$ UTR (Fig. 3e). For TOUCH3 (At2g41100), encoding the calcium-binding EF hand family protein $\mathrm{TCH} 3$, an XL site was identified in the first intron within the 5'UTR (Fig. 3f). 

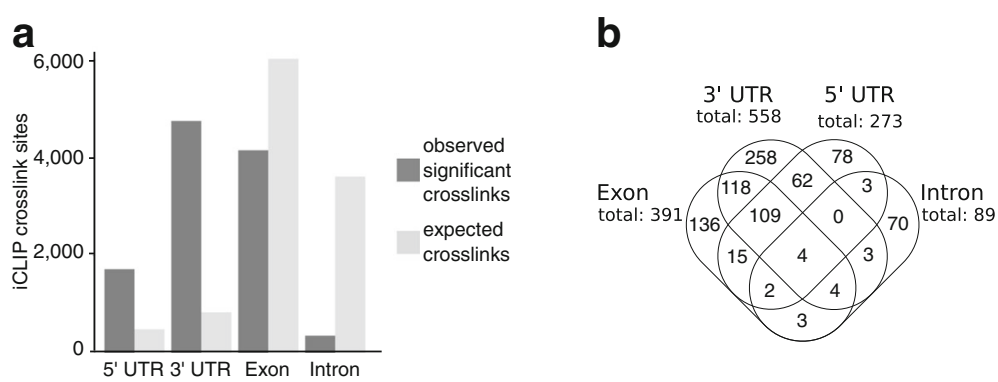

Fig. 2 Distribution of the AtGRP7 crosslink sites within the transcripts. a Number of significant crosslink sites (FDR $<0.05)$ in different transcript regions (dark grey bars) compared to a uniform distribution which would be expected according to the cumulative length of the indicated region in the genome based on TAIR10 (light grey bars). In all transcript regions a significant difference ( $p<0.001$, hypergeometric) could be observed between the number of observed significant crosslink sites and the number expected for a random distribution according to the size of the region. $\mathbf{b}$ Venn diagram showing the distribution of the crosslink sites between the different transcript regions. Numbers outside the Venn diagram state the overall number of transcripts with crosslink sites in the respective regions

\section{Confirmation of high-confidence binders among iCLIP targets by RIP-seq}

By adapting iCLIP to Arabidopsis plants subjected to UV-C crosslinking, we identified 858 candidate AtGRP7 target transcripts (Additional file 2: Table S2). However, iCLIP targets are biased towards transcripts with high expression levels, as previously shown for the heterogeneous nuclear ribonucleoprotein hnRNPH1 in HeLa cells [31]. This effect might be attributed to the low crosslinking efficiency upon UV treatment [32]. For example, it has been found that protein-RNA crosslinking occurs on only a minority of contact sites so that crosslinking efficiency can be as low as $1-5 \%$ for certain proteins [33]. This contrasts with more efficient formaldehyde crosslinking used in RIP [32]. To compare the spectrum of AtGRP7 target transcripts identified by these two crosslinking methods and to define a set of high-confidence binders of AtGRP7, we also performed RIP-seq experiments. AtGRP7:AtGRP7-GFP grp7-1 plants grown under the same conditions as for iCLIP were subjected to formaldehyde fixation at LL36. AtGRP7-GFP and associated RNAs were precipitated with GFP Trap beads and libraries were constructed for sequencing. The read statistics are shown in Additional file 2: Table S3.

To identify transcripts bound to AtGRP7-GFP (RIPseq targets) we determined the enrichment of transcripts co-precipitating with AtGRP7-GFP in the RIP-seq relative to the respective transcript level in poly(A)-containing RNA at LL36, as previously done for the RBPs Wig-1 in human cells and Imp in Drosophila [34, 35]. Transcripts with TPM (transcripts per million) $<5$ in the RIP-seq libraries were excluded from the analysis, leaving 3602 transcripts. Of those, 2453 showed a $\log _{2}$ fold enrichment $>0.5$ and $\mathrm{q}<0.001$ relative to poly(A)-containing RNA, determined by RNA-seq of Col-2 wild type (wt) in three biological replicates, and were therefore considered RIP-seq targets (Additional file 2: Table S4).
Using this filtering process, a suite of transcripts were recovered that included the previously identified in vivo targets AtGRP7, AtGRP8, an Aly/Ref related RNA binding protein/export factor (At5g59950), and a mitochondrial transcription termination factor family protein (At2g36000) [16]. At the same time, transcripts previously shown not to be bound by AtGRP7 but to be regulated indirectly, such as $A F C 2$ (encoding a LAMMER-type protein kinase [16]) or the PATHOGENESIS RELATED1 (PR1) transcript [36], were not among the RIP-seq targets, assuring a valid choice of the parameters.

When plotting the average of logarithmic normalized mean counts of each transcript in the RNA-seq data set versus transcripts in the RIP-seq data, we saw an overall positive correlation between RNA-seq counts and RIPseq counts (Additional file 1: Figure S5a). Nevertheless, many highly expressed genes were not represented in the RIP samples, suggesting that we did not just precipitate highly abundant transcripts nonspecifically. In turn, among transcripts with a high enrichment in RIP-seq were transcripts with a low read coverage in the RNAseq samples. Thus, binding of AtGRP7 monitored by RIP-seq does not simply reflect the expression level of the binding substrate. Notably, the number of identified RIP-seq targets for AtGRP7 is in the same order of magnitude as the 4262 RIP-seq targets identified for the Arabidopsis serine/arginine-rich (SR)-like RBP SR45 [5].

To confirm a set of high-confidence binders among iCLIP and RIP-seq targets, we determined the overlap of both data sets (Additional file 2: Table S5); 452 (53\%) of the 858 iCLIP targets were also found by RIP-seq, providing an independent confirmation. Conversely, 22.65\% of the 2453 RIP-seq targets were also found in iCLIP, whereas 2001 targets were identified only by RIP-seq. Overall, by combining iCLIP with RIP-seq we were able to identify a set of 452 high-confidence binders of AtGRP7. In parallel, RIP-seq extends the set of AtGRP7 targets. 


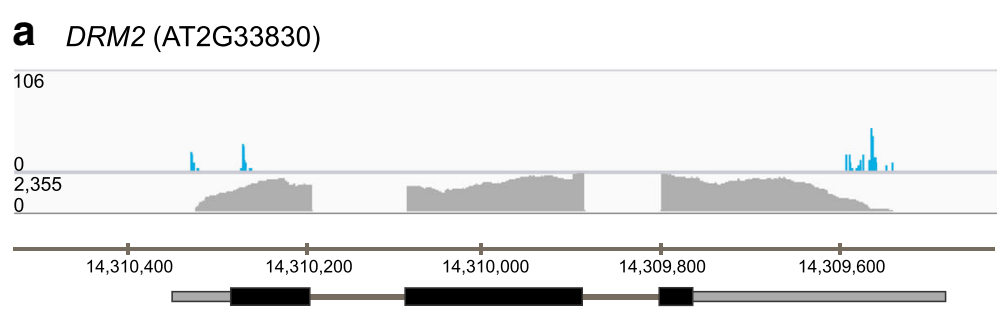

b $C C L$ (AT3G26740)

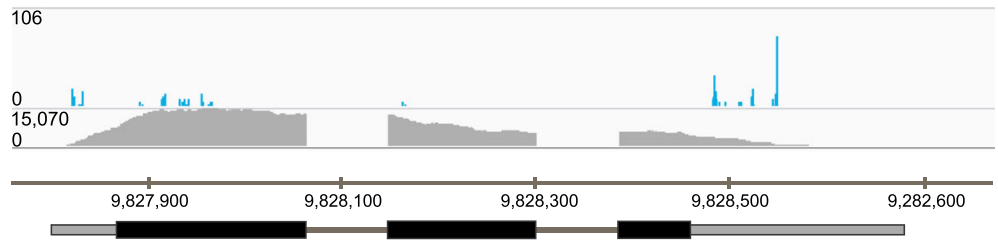

C AILP1 (AT5G19140)

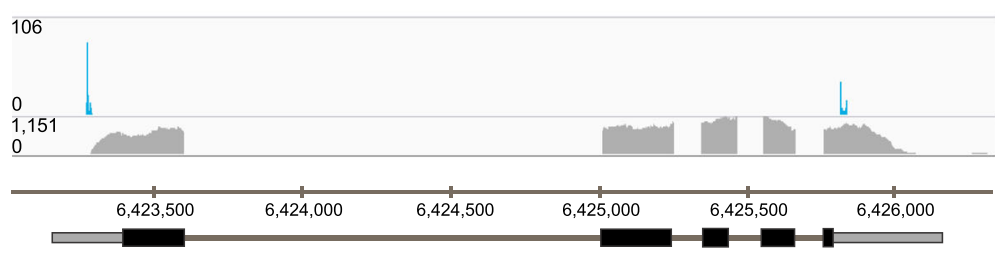

d HSPRO2 (AT2G40000)

106

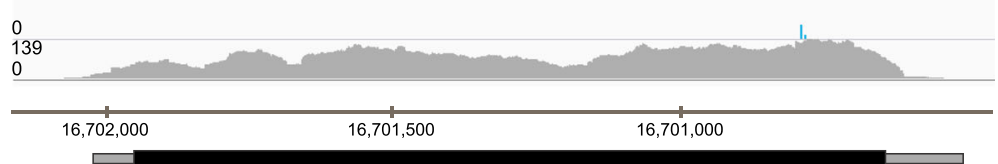

\section{e FNR2 (AT1G20020)}

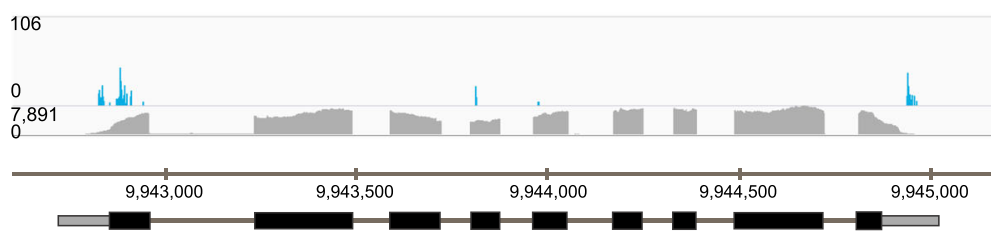

\section{f $T C H 3(\mathrm{AT} 2 \mathrm{G} 41100)$}

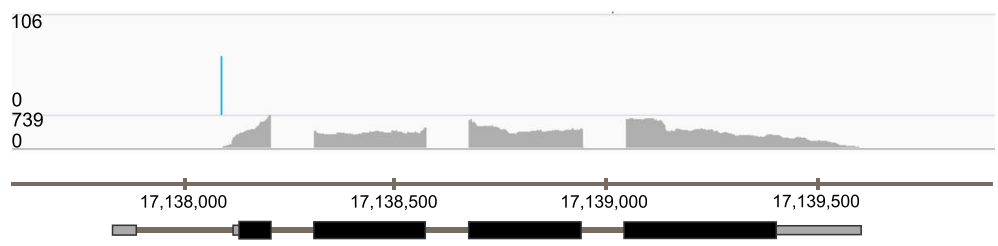

Fig. 3 Significant iCLIP crosslink sites on AtGRP7 target transcripts. Each panel shows the IGV genome browser tracks of significant crosslink sites determined in four out of five biological replicates at LL36 (top), the read counts in the LL36 RNA-seq (mean of three biological replicates; middle), and the representative gene model and chromosomal position (bottom). Thin bars represent 5' UTR (left) and 3' UTR (right); thick bars denote exons (coding sequences); and lines denote introns. The numbers on the $x$-axis refer to the chromosomal position. a DORMANCY/AUXIN ASSOCIATED FAMILY PROTEIN (DRM2); b CCR-LIKE (CCL); c ALUMINIUM-INDUCED-LIKE PROTEIN 1 (AILP1); d ORTHOLOG OF SUGAR BEET HS1 PRO-1 2 (HSPRO2); e FERREDOXIN NADP(H) OXIDOREDUCTASE 2 (FNR2); $\mathbf{f}$ TOUCH3 (TCH3) 
Validation of iCLIP and RIP-seq targets by RIP-qPCR

To obtain an independent validation of the targets identified by both iCLIP and RIP-seq, we performed RIPqPCR on AtGRP7::AtGRP7-GFP grp7-1 plants subjected to formaldehyde fixation in three biological replicates. Enrichment upon precipitation with GFP Trap beads but not upon mock precipitation with RFP Trap beads was confirmed for circadian clock-regulated transcripts, AtGRP7, DRM2, ATHSPRO2, the PSBP-1 transcript encoding the Photosystem II subunit P1 (At1g06680), an aluminum-induced transcript of unknown function (At3g15450), AILP1, FERRETIN1 (FER1; At5g01600), FATTY ACID DESATURASE 2 (FAD2), and the coldregulated transcripts COR15A (At2g42540), COR15B (At2g42530), COR413-PM1 (At2g15970), and KIN1 (At5g15960) (Fig. 4). The transcripts were barely detectable in precipitates from GFP-only plants, confirming low background noise in the control samples. As negative controls we chose PP2A and TOUGH (At5g23080), encoding an RBP involved in microRNA biogenesis, which were not detected by either iCLIP or RIP-seq. These transcripts were not enriched in RIP-qPCR.

This confirmation of targets suggests that the overlap between iCLIP and RIP-seq represents high-confidence in vivo targets of AtGRP7. Moreover, binding of transcripts encoding the transcription factors ETHYLENE RESPONSE FACTOR 4 (ERF4; At3g15210), WRKY33 (At2g38470), and SALT TOLERANCE ZINC FINGER (STZ; At1g27730), which were identified by RIP-seq only, was validated in independent biological replicates by RIP-qPCR. Their absence from the iCLIP targets could be due to the reduced crosslinking efficiency of UV light or because they represent indirect targets.

The circadian clock-regulated CCR-LIKE (CCL) transcript and PATHOGENESIS RELATED 4 (PR4), encoding a protein with similarity to the antifungal chitin-binding protein hevein from rubber tree latex, had been identified only by iCLIP (Additional file 2: Table S2). They were significantly
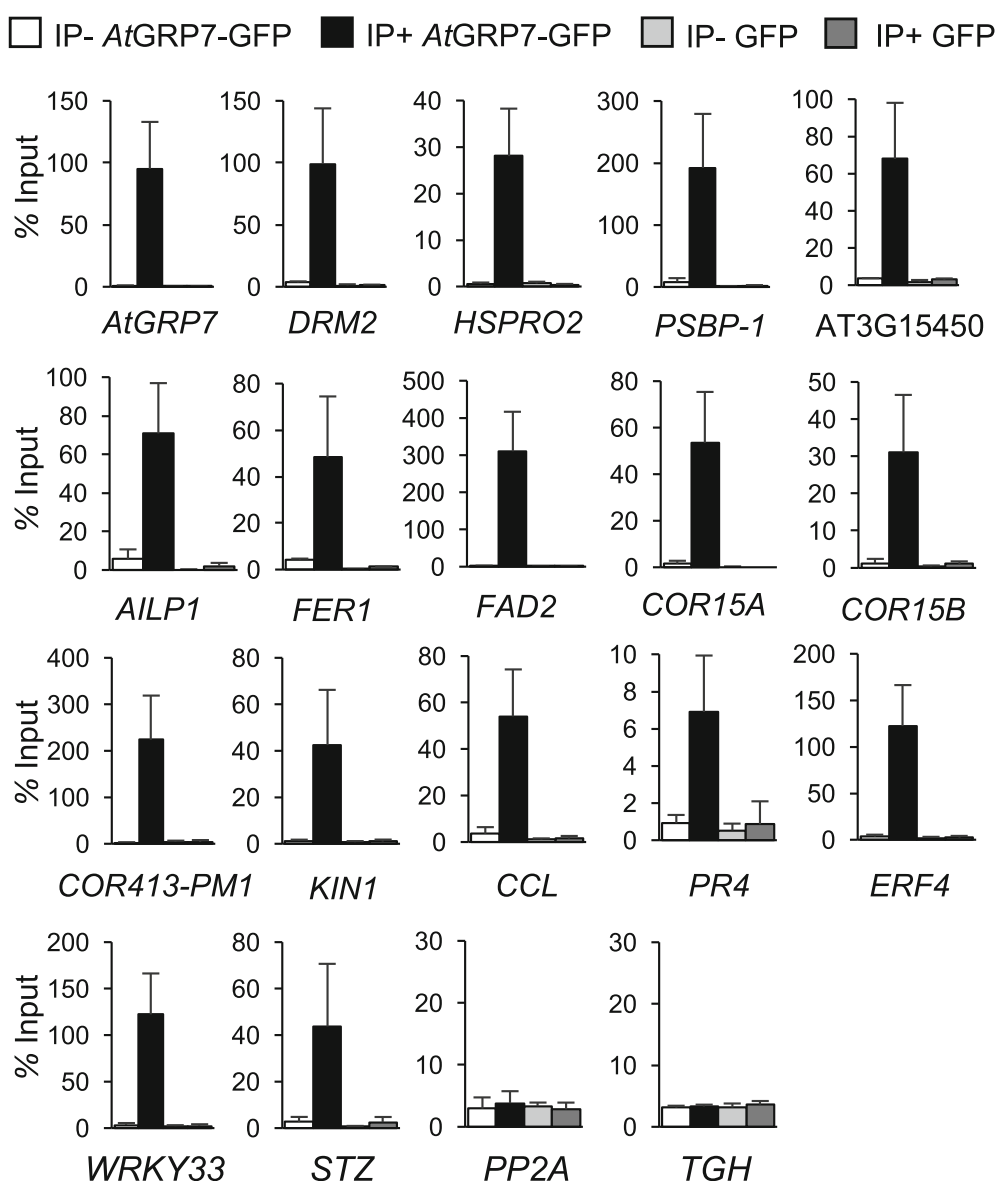

Fig. 4 Validation of candidate iCLIP and RIP-seq targets by RIP-qPCR. RIP-qPCR analysis of iCLIP and RIP targets that are circadianly regulated (AtGRP7, DRM2, HSPRO2, PSBP-1, AT3G15450, AlLP1, FER1, and FAD2) or cold regulated (COR15A, COR15B, COR413-PM1, and KIN1), iCLIP-only targets (CCL and PR4), and RIP-only targets (ERF4, WRKY33, and STZ) in AtGRP7::AtGRP7-GFP grp7-1 and AtGRP7::GFP-only plants. Transcript levels in the GFP Trap precipitate $(I P+)$ or RFP Trap precipitate (IP-) are presented relative to the transcript levels in the input. Data represent mean \pm standard deviation of three biological replicates. PP2A and TGH as unbound transcripts serve as negative controls 
enriched in RIP-qPCR with GFP Trap beads but not with RFP Trap beads. The confirmation by RIP-qPCR indicates that these transcripts can indeed be crosslinked to AtGRP7 by formaldehyde.

For some of the transcripts, the level of precipitated RNA seems higher than expected based on the input (Fig. 4). This observation is not unprecedented and may be due to different efficiencies in RNA extraction in immunoprecipitated fractions compared to total extract as well as a higher efficiency of reverse transcriptase on lower amounts of RNA [3, 37].

\section{Determination of the AtGRP7 binding landscape}

A key advantage of iCLIP is its precise mapping of the interaction site, thereby allowing the prediction of a binding motif. Consequently, the next step was the investigation of candidate AtGRP7 binding motifs in the vicinity of the crosslink sites. The presence of XL sites in $5^{\prime}$ UTRs, exons, introns, and 3' UTRs could imply different functions of the protein on these regions. Thus, motifs were generated for each transcript region separately.

The genomic sequence at the XL sites was extended by ten nucleotides in either direction and used to identify conserved motifs with the MEME-Suite [38]. To discriminate against random binding events, a background was generated by simulating the identical number of crosslinks uniformly in the corresponding $5^{\prime}$ UTR, exon, introns, and 3' UTR, respectively. The simulated XL sites were extended and genomic sequences extracted, exactly as for the observed XL sites. The resulting significant motifs were generally U/C-rich. One significant motif was obtained in the exons, introns, and 3' UTR, respectively, and two for the 5' UTR (Fig. 5). To perceive differences between the motifs identified by MEME, we performed a clustering analysis based on pairwise comparison between motifs across regions using the $\mathrm{R}$ package DiffLogo (Additional file 1: Figure S6a). In the resulting distance tree, the exon and 5' UTR motifs clustered together and consequently were closely related. In contrast, the 3 ' UTR motif is located outside this subgroup and therefore exhibited the largest difference from the other motifs. This may indicate a variation in binding preference for AtGRP7 between the transcript regions and point to different functions of the protein depending on the region.

In a complementary approach, we determined enriched pentamers in the vicinity of the XL sites essentially as previously described [10]. Again, the XL site was extended by ten nucleotides in either direction. A five-nucleotideframe was shifted along these sequences, all pentamers within these frames were recorded, and enriched pentamers were calculated with a Z-score analysis. For the $5^{\prime}$ UTR and the exon, the evaluation of pentamer frequencies around the $\mathrm{XL}$ sites also yielded U/C-rich sequences (Additional file 2: Table S6). Similar to the MEME analysis, the pentamers in the 3' UTRs were U-rich as well, but the pentamer analysis revealed a higher frequency of $G$ than the MEME motif. For introns, the pentamer frequencies differed slightly from the MEME results, being more C/G-rich.

So far, in vitro binding requirements of recombinantly expressed AtGRP7 have been investigated for the 3' UTR and intron of its own transcript, using electrophoretic mobility shift assays and fluorescence correlation spectroscopy $[14,18,39]$. For the 3' UTR, iCLIP now identified three significant crosslink sites around a 32-nucleotide sequence previously used for binding studies (Additional file 1: Figure S7a). Notably, upon scanning the AtGRP7 sequence for the significant $3^{\prime}$ UTR motif using FIMO [38], several matches were found within or close to the binding region defined in vitro. Furthermore, sequences corresponding to the pentamers enriched in the $3^{\prime}$ UTR were found in this 32-nucleotide region, including one that overlapped a minimal AtGRP7 binding sequence delineated by a deletion analysis [39]. Thus, a known in vitro binding site was confirmed in vivo.

Furthermore, the iCLIP data revealed AtGRP7 binding to the first half of the intron located upstream of the cryptic $5^{\prime}$ splice site. Several matches of the MEME intron motif were observed near the XL sites (Additional file 1: Figure S7b). An additional AtGRP7 binding site had been determined within the second half of the intron in vitro $[14,40]$. No significant XL sites were obtained in this

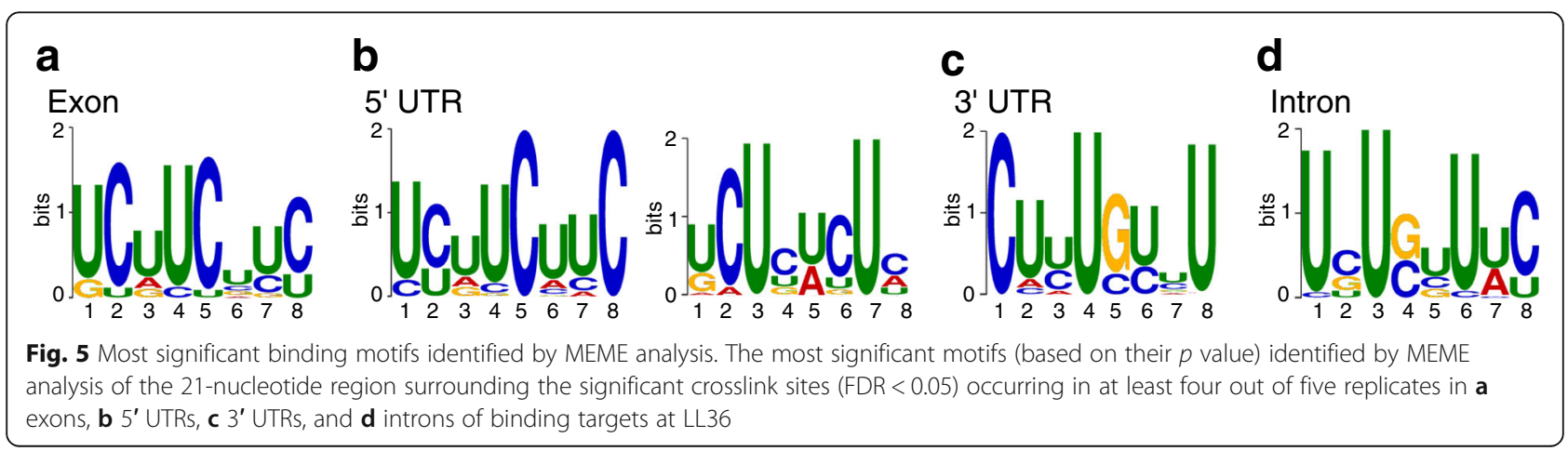


region. However, an intron motif was detected next to the determined in vitro binding site [40]. This binding site may not be accessible for AtGRP7 in vivo under the conditions analyzed.

\section{Impact of AtGRP7 on candidate targets}

Identification of in vivo targets represents a first step towards understanding posttranscriptional networks controlled by RBPs. The consequences the binding may have for the transcriptome are usually assessed by knocking down the RBP and monitoring the fate of the target mRNAs [41-43]. To unravel whether the in vivo targets we have identified are regulated by AtGRP7 at the RNA level, RNA-seq was performed on a loss-of-function mutant. Because the grp7-1 T-DNA line has elevated AtGRP8 levels due to relief of repression by $A t \mathrm{GRP} 7$, we used the grp7-1 $8 i$ line that has an RNAi construct against AtGRP8 and expresses AtGRP8 at levels comparable to wt plants [21]. As the redundancy between AtGRP7 and AtGRP8 may mask a clear loss-of-function phenotype, we included plants with constitutively elevated AtGRP7 levels (AtGRP7-ox) in the analysis. Libraries were prepared from plants harvested in parallel to the samples used for RIPseq at LL36. The read statistics are presented in Additional file 2: Table S7. Only transcripts with a TPM > 1 in at least one of the genotypes (average of three biological replicates) were considered. Transcripts with $\mathrm{q}<$ 0.05 were considered significantly differentially expressed either between mutant and wt, or between AtGRP7-ox plants and wt (differentially expressed genes (DEGs)). The list of DEGs was cross-referenced against the iCLIP and RIP-seq targets (Additional file 1: Figure S8a, b; Additional file 2: Table S8). Of the iCLIP targets, 93 were significantly differentially expressed in grp7-1 8i (of 2087 DEGs) and 534 in AtGRP7-ox plants (of 7855 DEGs); 62 transcripts were DEGs in both genotypes. When considering the highconfidence binders that were also identified by RIP-seq, 58 were significantly differentially expressed in grp7-1 8i, 293 in AtGRP7-ox, and 38 in both genotypes. For targets identified by RIP-seq, 365 were DEGs in grp7-1 8i, 1207 in AtGRP7-ox, and 157 in both genotypes. The considerably lower number of DEGs in the loss-of-function mutant than in the AtGRP7-ox plants is at least partly due to the redundancy of $A t \mathrm{GRP7}$ and $A t \mathrm{GRP} 8$, as seen before [16, 44].

Overall, a similar proportion of transcripts were either upregulated or downregulated in AtGRP7-ox plants or the grp7-1 8i mutant, respectively (Fig. 6a, e). Notably, significantly more of the differentially expressed iCLIP targets were downregulated in the AtGRP7-ox plants than upregulated (Fig. 6b). This was also seen for the RIP-seq-only targets or the high-confidence binders identified by both iCLIP and RIP-seq (Fig. 6c, d). In contrast, the differentially expressed iCLIP targets, RIP-seq targets, and high-confidence binders were preferentially upregulated in the $\operatorname{grp} 7-18 i$ line (Fig. $6 \mathrm{f}-\mathrm{h}$ ). This indicates that DEGs bound in vivo by AtGRP7 are mostly under negative control by AtGRP7. In contrast, some of the genes that are positively regulated by AtGRP7 may be controlled indirectly via intermediate factors. For example, the defense-related $P R 1$ transcript is upregulated by elevated AtGRP7 levels through NPR1dependent activation of the promoter, and accordingly, the PR1 transcript is not an in vivo target [36].

The differential expression of high-confidence binders was validated by RT-qPCR in independent samples (Additional file 1: Figure S8c). Considering the involvement of AtGRP7 in the response to diverse stress factors, we selected candidate targets associated with stress responses. The cold-responsive COR15A transcript encoding a chloroplast protein of unknown function was reduced in AtGRP7-ox plants. FAD2 encoding an ERlocalized $\omega 6$ desaturase required for salt tolerance was also reduced [45]. The level of the clock-regulated DRM2 was reduced, as was FER1. ATHSPRO2 was elevated in grp7-1 $8 i$ compared to wt. Furthermore, a suite of transcription factors of the AP2/ETHYLENE RESPONSE FACTOR and WRKY families, which were only identified by RIP-seq, were expressed at higher levels in grp7-1 8i compared to wt (Additional file 1: Figure S8d). Among those were ERF4, which is associated with jasmonic acid, ethylene, and abscisic acid signaling, and the zinc finger factor $S T Z$, which plays both a positive and negative role in the tolerance of plants to salinity, heat, and osmotic stress [46]. Furthermore, WRKY33, which regulates the antagonistic relationship between defense pathways mediating responses to Pseudomonas syringae and necrotrophic fungal pathogens, respectively, is elevated in grp7-1 $8 i$. It remains to be determined whether in vivo binding of AtGRP7 to HSPRO2 or WRKY33 and the differential expression of these genes relates to the role of AtGRP7 in plant innate immunity [19, 36, 47, 48]. Overall, the differential expression of the selected DEGs measured by RT-qPCR correlated well with the $\log _{2}$ fold change in the RNA-seq data (Additional file 1: Figure S8c, d).

\section{AtGRP7 regulates circadian target transcripts}

Among the AtGRP7 iCLIP and RIP targets was the circadian clock regulated AILP1, previously identified by fluorescent differential display as aberrantly expressed upon AtGRP7 overexpression [18]. To determine the impact of AtGRP7 on clock-regulated transcripts globally, the targets were compared to a list compiling 5230 circadianly regulated Arabidopsis transcripts, presented in [49]. Indeed, 205 of the 452 high-confidence binders (45\%), 383 of the 858 iCLIP targets (45\%), and 924 of the 2453 RIP targets at LL36 (38\%) were circadianly regulated according to [49]. In contrast, of all the genes expressed at LL36 in our RNA-seq data, only $23 \%$ were circadianly regulated according to [49]. 

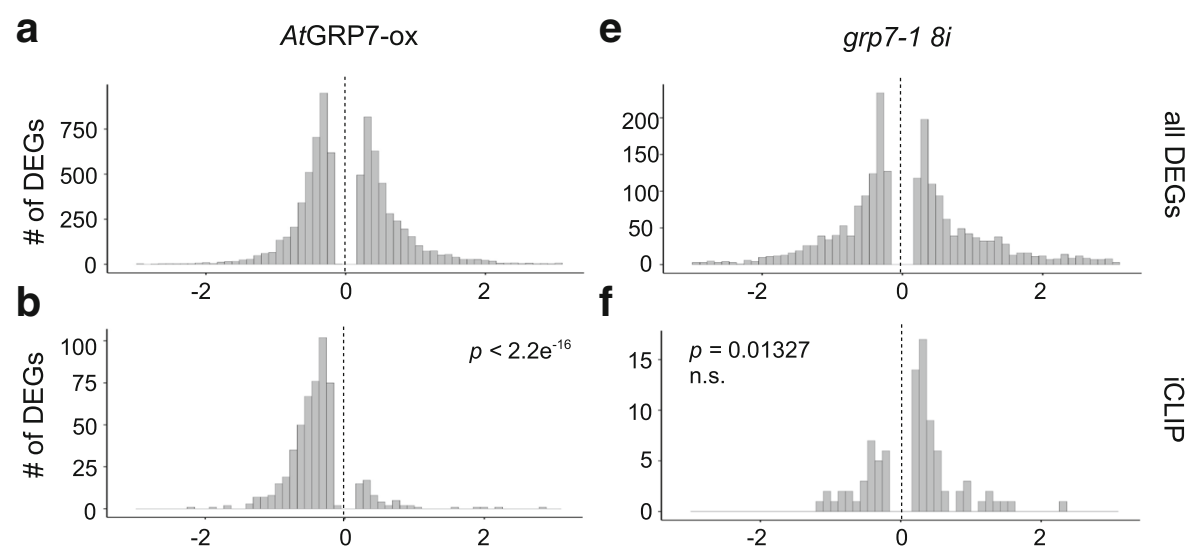

$\overline{\bar{\rho}}$
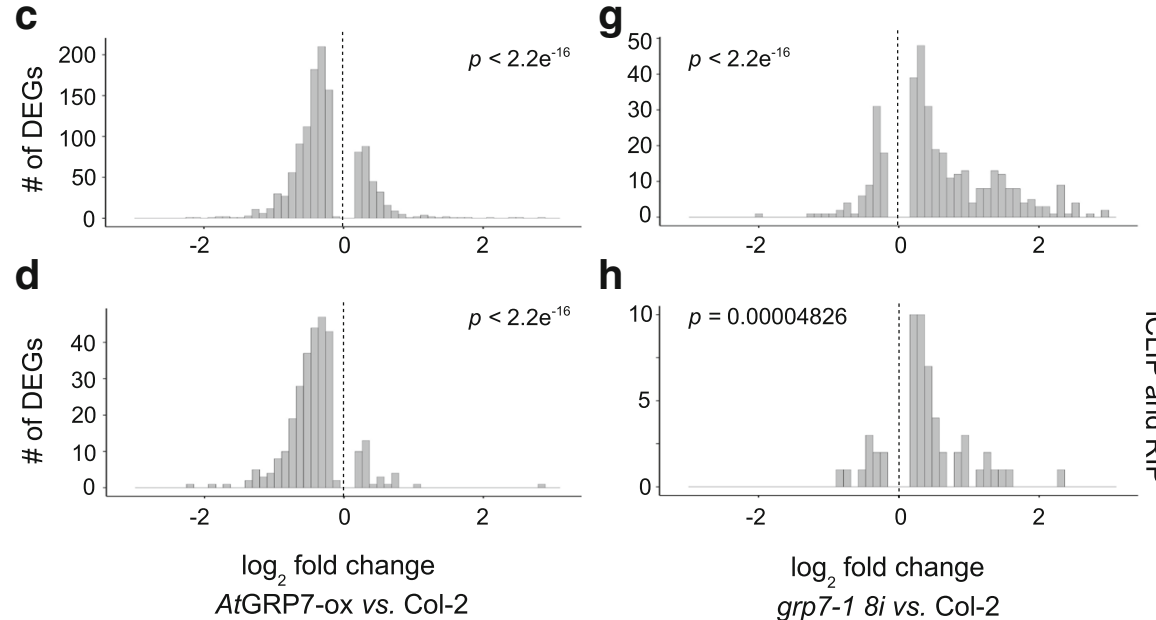

h

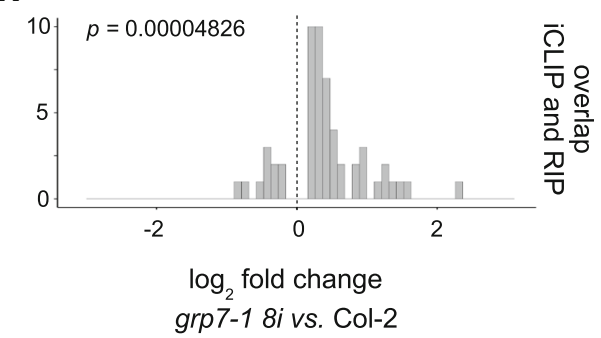

Fig. 6 Changes in distribution of the $\log _{2}$ fold changes of genes differentially expressed at LL36 (DEGs) in the grp7-1 8i mutant or AtGRP7-ox plants upon binding to AtGRP7. Log2 fold change distribution of all genes differentially expressed at LL36 (DEGs) from the RNA-seq data set (a AtGRP7-ox, e grp7-1 8i), as well as of iCLIP targets with a significant differential expression (b AtGRP7-ox, $\mathbf{f}$ grp7-1 8i), RIP targets with a significant differential expression (c AtGRP7-ox, g grp7-1 8i), and high-confidence binders identified by both iCLIP and RIP (d AtGRP7-ox, h grp7-1 8i). The distribution of all identified DEGs in RNA-seq $(\mathbf{a}, \mathbf{e})$ was tested pairwise against all target groups. The resulting $p$ value is displayed accordingly

Therefore, we monitored the circadian expression patterns of iCLIP targets differentially expressed in the AtGRP7-ox plants with high temporal resolution. Plants grown in $12 \mathrm{~h}$ light-12 $\mathrm{h}$ dark cycles were transferred to constant light and harvested at 2-h intervals, starting at LL20. CCL and DRM2 showed a reduced peak in two independent transgenic AtGRP7-ox lines on three consecutive days, indicating that AtGRP7 indeed negatively regulates the oscillations of these transcripts. Although one would expect an opposite phenotype in the grp 7-1 $8 i$ line, the oscillations were similar to wt plants (Fig. 7), suggesting AtGRP7 acts redundantly with other factors in shaping these transcript oscillations.

\section{AtGRP7 targets in the subjective morning}

To test whether the spectrum of AtGRP7 targets depends on the time of the day, we performed another iCLIP experiment on AtGRP7::AtGRP7-GFP grp7-1 plants harvested $12 \mathrm{~h}$ out of phase, at LL24. The read statistics are presented in Additional file 2: Table S9. After processing the reads as done for the LL36 iCLIP targets, we arrived at 469 transcripts with significant XL sites in at least two of the three AtGRP7::AtGRP7-GFP grp7-1 replicates and absent in the GFP-only plants and AtGRP7::AtGRP7 $R^{49} Q$-GFP plants (Additional file 2: Table S10). A similar distribution of XL sites in the different regions of the transcript was found as for the transcripts bound in the subjective evening, at LL36 (Additional file 1: Figure S9b). After normalizing to the length of the transcript region, the highest number of XL sites again was found in the 3' UTRs (Additional file 1: Figure S9a).

Of the transcripts identified in iCLIP, 386 were bound at both time points. The distribution of XL sites was similar at LL24 and LL36, e.g., for KIN1, COR27, COR413-PM1, or FNR2 (cf. Additional file 1: Figure S4). Other examples were NITRATE REDUCTASE 2 (NIA2; At1g37130), where LL24 and LL36 share a similar XL site in the 3' UTR, and POLY A BINDING PROTEIN 2 (PABP2; At4g34110), which 

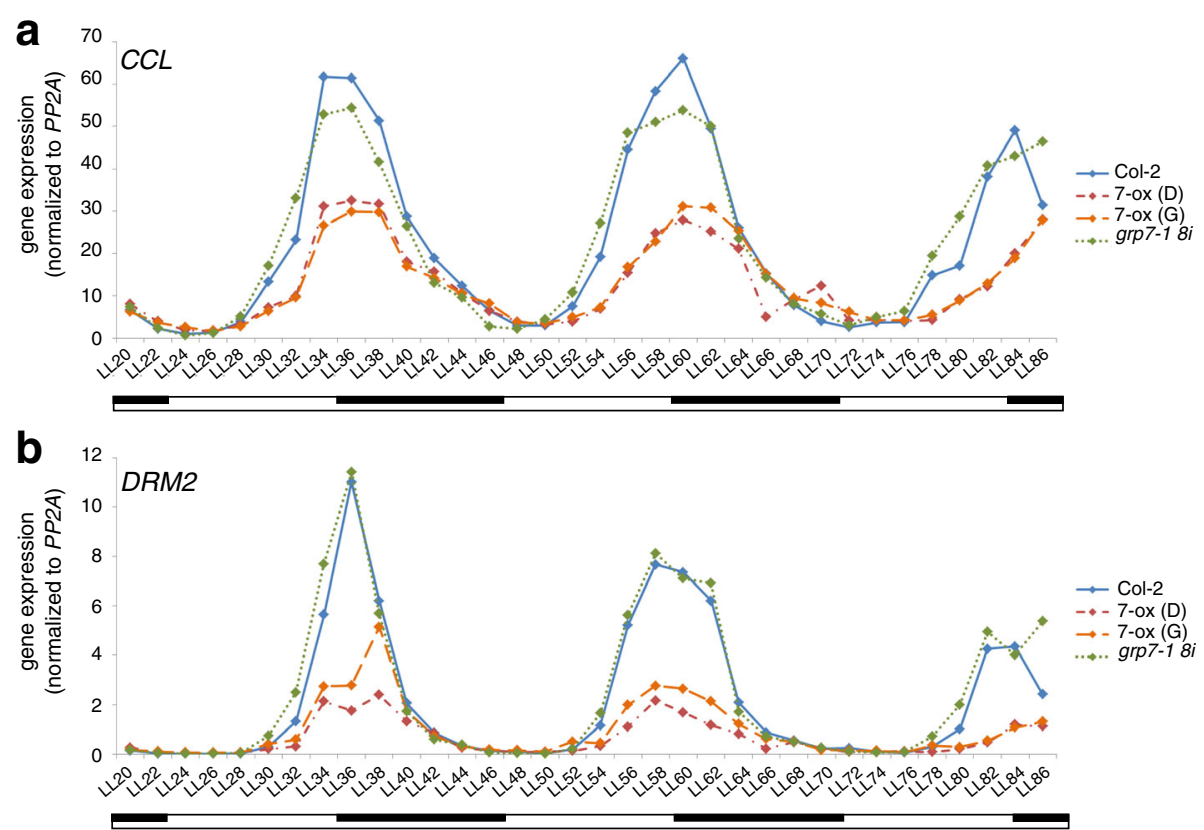

Fig. 7 Circadian expression of CCL and DRM2 measured in 2-h time intervals. Seedlings were grown in 12-h light and 12 -h dark phase for 16 days and then transferred to constant light (LL). Transcript levels of a CCL (AT3G26740) and b DRM2 (AT2G33830) were determined every $2 \mathrm{~h}$ in wt (Col-2), two independent AtGRP7 overexpressing lines (7-ox), and the grp7-1 8i mutant. The time course was performed twice with similar results. Open bar, constant light; inserted dark bar, subjective night

contains a group of XL sites in the 5' UTR (Additional file 1: Figure S9c, d).

As done for LL36, enriched motifs in the vicinity of XL sites were determined by MEME in the LL24 targets (Additional file 1: Figure S10). As seen before, the significant motifs for the exon and the $5^{\prime}$ UTR are U/C rich. When clustering the motifs of both time points with DiffLogo [50], the LL24 and LL36 motifs of these regions were grouped together (Additional file 1: Figure S6b). Similarly, the motifs for the intron at both time points were more closely related to each other than to the other motifs of the same time point. The same effect was observed for the 3' UTR, indicating that the bound motifs in the different transcript regions were highly similar for the subjective morning and the subjective evening.

Independent validation of the iCLIP targets was performed by RIP-seq of AtGRP7::AtGRP7-GFP grp7-1 plants subjected to formaldehyde fixation at LL24. The read statistics are displayed in Additional file 2: Table S11. Of the 2256 RIP-seq targets with a $\log _{2}$ fold enrichment $>0.5$ and $\mathrm{q}<0.001$ over poly(A) RNA (Additional file 2: Table S12), 196 targets were identified also by iCLIP (Additional file 2: Table S13). Thus, $42 \%$ of the 469 iCLIP targets were also identified by RIP and, conversely, $8.7 \%$ of the 2256 RIPseq targets were also identified by iCLIP.

To unravel an impact of AtGRP7 on the LL24 targets, we cross-referenced the targets against RNA-seq data generated in grp7-1 $8 i$ and AtGRP7-ox plants harvested at
LL24. The read statistics are shown in Additional file 2: Table S14. Again, transcripts with $\mathrm{q}<0.05$ were considered significant DEGs between either mutant and wt or AtGRP7-ox plants and wt (Additional file 2: Table S15; Additional file 1: Figure S11). Of the iCLIP targets, 24 were significantly differentially expressed in grp7-1 $8 i$ (of 731 total DEGs in the mutant), and 306 in AtGRP7-ox plants (of 5927). Eighteen transcripts were DEGs in both genotypes. When considering the high-confidence binders that were also identified by RIP-seq, 14 were DEGs in grp7-1 8i, 147 in AtGRP7-ox plants, and nine in both. For targets identified by RIP-seq, 177 were DEGs in grp7-1 8i, 996 in AtGRP7-ox, and 51 in both. As observed for LL36, DEGs bound in vivo by AtGRP7 are mostly under negative control by AtGRP7 at LL24 (Additional file 1: Figure S12).

Similar to LL36, the binding targets at LL24 were enriched for circadianly regulated transcripts, as listed in [49]: 97 of the 196 high confidence binders (49\%), 231 of the 469 iCLIP targets (49\%), and 876 of the 2256 RIP targets (39\%) at LL24 are circadianly regulated. In contrast, only $23 \%$ of all transcripts expressed at LL24 in our RNA-seq were circadianly regulated.

Alternative splicing targets identified by iCLIP and/or RIP-seq Previously, we identified 59 alternative splicing events significantly changed in response to elevated AtGRP7 levels [16]. To monitor splicing changes globally, we analyzed the RNA-seq data of the grp7-1 8i mutant and 
AtGRP7-ox plants using SUPPA [51]. SUPPA determines the percent spliced in (PSI) value, defined as the ratio between the TPM of the isoform including the event and the overall TPM of all isoforms for single splicing events. Changes of more than $10 \%$ in the isoform ratio $(|\Delta \mathrm{PSI}|>$ 0.1 ) between the grp7-1 $8 i$ mutant and wt or AtGRP7ox and wt, respectively, with $p<0.01$ were considered significant (Additional file 2: Table S16). Selected events were validated by RT-PCR.

The most common type of alternative splicing events affected in both genotypes was intron retention. The transcript encoding the calcium-binding EF hand family protein $\mathrm{TCH} 3$ was found in both RIP and iCLIP at LL36. In the grp7-1 $8 i$ mutant, more intron retention in the first exon was detected (Additional file 1: Figure S13a). The location of 5' UTR XL sites close to the intron retention event suggests an effect of AtGRP7 binding on the splicing event. For the iCLIP target FNR2 the first intron, which contains a PTC, was retained more often in AtGRP7-ox plants than in the wt and the XL site mapped in close proximity of the event (Additional file 1 : Figure S13b). RIP-RT-PCR using primers that detect both isoforms showed that AtGRP7 preferentially binds to the spliced isoform (Additional file 1: Figure S14). The At1g28580 transcript encoding a GDSL-like lipase was identified by RIP-seq at LL36. Elevated AtGRP7 levels promote intron retention in the first exon, also leading to a PTC. The increase in the intron retained band was confirmed (Additional file 1: Figure S13c). At5g66240 encodes a Transducin/WD40 repeat-like superfamily protein and was identified by RIP-seq at LL36. An increase in AtGRP7 levels leads to both an increased steadystate abundance and less retention of intron 1 , predicted to remove three amino acids (Additional file 1: Figure S13d). RIP-RT-PCR showed that AtGRP7 binds to both isoforms (Additional file 1: Figure S14). At3g17100 encodes the transcription factor ATBS1 INTERACTING 3 (AIF3), detected by RIP-seq at both time points. Increased AtGRP7 levels led to retention of intron 1 in the $5^{\prime}$ UTR (Additional file 1 : Figure S13E). RIP-RT-PCR showed that AtGRP7 binds to the small isoform (Additional file 1: Figure S14).

Another splicing event, exon skipping, was also found among the iCLIP and RIP-seq targets. An example for a RIP-seq target is FAX4 (At1g33265), which encodes a fatty acid exporter in the chloroplast membrane. Upon AtGRP7 overexpression, exon 2 skipping was promoted (Additional file 1: Figure S13f), leading to a frameshift and a PTC, which causes the predicted protein to lose a polyleucine stretch and three transmembrane helices. RIP-RT-PCRs confirmed that AtGRP7 interacts at least with the longer isoform, which retains the alternative exon (Additional file 1: Figure S14).

Furthermore, we found alternative usage of $5^{\prime}$ or $3^{\prime}$ splice sites among the AtGRP7 targets. The RIP-seq target At1g76020 encodes a thioredoxin superfamily protein, where an elevated AtGRP7 dosage leads to a shift to an alternative $5^{\prime}$ splice site and the retention of 88 nucleotides of the first intron (Additional file 1: Figure S13g). Again, the event introduces a PTC and the isoform is a predicted NMD target [52]. RIP-RT-PCR showed that AtGRP7 binds to the fully spliced isoform (Additional file 1: Figure S14). For all these splicing events the isoform ratio in wt was similar at LL24 and LL36, indicating that these events are not under circadian control (Additional file 2: Table S17). At LL24, mis-expression of AtGRP7 had a similar impact on these splicing events as observed at LL36 (Additional file 1: Figure S15; Additional file 2: Table S17). Only for $T C H 3$ was the increased intron retention in the mutant not statistically significant at LL24 in contrast to LL36. This indicates that the effect of AtGRP7 on these investigated alternative splicing events in bound targets was not timeof-day dependent.

Several transcripts with splicing events previously shown to be regulated by AtGRP7 were also identified here [16] In addition to AtGRP7 and AtGRP8, At2g36000, encoding a mitochondrial termination factor family protein with an intron in its 3' UTR that is spliced in a temperaturedependent manner [53], the Aly/Ref related RNA binding protein/export factor (At5g59950), the chaperone DnaJ (At3g62190), the ankyrin repeat-containing protein AKR2 (At4g35450), and the cofactor for nitrate reductase CNX7 (At4g10100) were found by RIP-seq.

As SUPPA does not reveal the differential usage of polyadenylation sites, we analyzed selected examples separately with RT-PCR. At1g45474, encoding the LHCA5 protein of the light harvesting complex of photosystem 1, was among the RIP-seq targets and combines an alternative $5^{\prime}$ splice site with skipping of the last exon, leading to an alternative 3' UTR. Both, the wt and grp7-1 8i showed a preference for the distal polyadenylation site, whereas in AtGRP7-ox plants the distal and proximal polyadenylation sites were used similarly (Additional file 1: Figure S13h). The thiamine biosynthetic enzyme THIAMIN C (THIC; At2g29630), an iCLIP and RIP-seq target, is known to undergo alternative polyadenylation as well. Similar to LHCA5, the event combines an alternative $5^{\prime}$ splice site with skipping of the last exon. Additionally, THIC harbors a Thiamin-dependent riboswitch in the 3' UTR [54]. An increasing AtGRP7 level influences alternative splicing of intron 6, which leads to a decrease of the ID9 isoform polyadenylated at the distal site (Additional file 1: Figure S13i). This isoform contains the majority of the riboswitch, without the initial eight nucleotides, and is known to be less stable. In contrast, isoform 1 is more stable, lacks the riboswitch, and leads to proximal polyadenylation. AtGRP7 binds to both isoforms (Additional file 1: Figure S14). Overall, these show that AtGRP7 impacts different types of splicing events by direct in vivo binding to the targets and that it can affect alternative polyadenylation through its impact on splicing of the penultimate exon. 


\section{Discussion}

Here we present the first iCLIP analysis identifying in vivo targets of plant RBPs and their binding landscape at a genome-wide scale. For the circadian clock regulated RBP AtGRP7 we identify 858 transcripts with significant crosslink sites present at the same position in at least four out of five biological replicates of UV crosslinked AtGRP7::AtGRP7-GFP grp7-1 plants, and absent in plants expressing GFP-only or an RNA-binding-dead variant of AtGRP7. Of these iCLIP targets, 53\% were also independently identified by RIP-seq, and thus represent a set of high-confidence binders.

\section{Detection of iCLIP targets and discrimination against background}

iCLIP has become a state-of-the-art method to study RNA-protein interactions in vivo; to date, however, the successful application of iCLIP in Arabidopsis has not been reported. iCLIP critically relies on UV-C crosslinking. Thus, we first tested this step and its effect on Arabidopsis seedlings, as it was not clear whether only transcripts present in the top cell layers of the leaf would be crosslinked. The UV dose of $500 \mathrm{~mJ} / \mathrm{cm}^{2}$ used in our experiments is in the same range as the 100 or $150 \mathrm{~mJ} / \mathrm{cm}^{2}$ used in mammalian cells [10, 55, 56], $250 \mathrm{~mJ} / \mathrm{cm}^{2}$ used in Drosophila [35], and $400 \mathrm{~mJ} / \mathrm{cm}^{2}$ used in neuronal cells [43].

We found that irradiated leaves eventually bleached, indicating that the UV light indeed reaches the interior of the leaves. The emergence of new leaves several days after irradiation may indicate that the UV light does not reach deeply into the meristem and thus transcripts expressed there may not be crosslinked efficiently. Additionally, we compared the iCLIP targets with transcripts that have been shown to be preferentially expressed in the mesophyll or the vasculature of 9-day-old seedlings based on a more than tenfold enrichment in these tissues compared to the average transcript level in total leaves [57]. Of 250 transcripts preferentially expressed in the mesophyll, 51 were recovered by AtGRP7 iCLIP at LL36, with 21 of those also found by RIP-seq. Of the 280 transcripts preferentially expressed in the vasculature, three were found by iCLIP and two also by RIP-seq. Among the iCLIP targets we also find plastidic carboanhydrase CA1 (At3g01500), established as a marker for mesophyll cells [58]. The identification of transcripts in these tissue layers indicates that the UV treatment crosslinks transcripts in the interior of the leaf.

On the other hand, transcript levels of UV stress response marker were not elevated in the timeframe relevant for the experimental procedure and thus the physiological state of the plants should not be grossly altered. It cannot be ruled out that posttranslational modifications, e.g., in response to UV-activated kinase signaling, impacts the RNA binding properties of RBPs, a limitation inherent in all CLIP studies. However, UV crosslinking occurs on a very fast time scale, and any modification occurring after the formation of the covalent bonds does not influence the spectrum of targets. Recently, irradiation with $254 \mathrm{~nm}$ UV light has also proven successful for crosslinking mRNAs and bound proteins in studies aimed at identifying all mRNA interacting proteins in Arabidopsis cell cultures, protoplasts, leaves, or etiolated seedlings [59-61].

We used the strategy developed by König et al. [10] to determine the XL sites, where significant XL sites were discriminated against a randomly generated background using FDR. To select the most reliable targets, we applied a stringent filtering criterion, requiring that the significant XL sites were called in at least four out of five independent biological replicates. When we increased the stringency and considered XL sites present at the same position in all five replicates, some of the known and confirmed AtGRP7 targets were not present any more. As RNA-binding domains contact more than one nucleotide in the RNA, it seems plausible that the XL sites of the different replicates disperse and do not necessarily map to the identical nucleotide. Requiring the $\mathrm{XL}$ sites of all five replicates to map to the exact same position eliminates those sites that might scatter around a few nucleotides.

Furthermore, we excluded transcripts from the analysis that contained significant $\mathrm{XL}$ sites in plants expressing either GFP alone or AtGRP7 $\mathrm{R}^{49} \mathrm{Q}-\mathrm{GFP}$ under control of the AtGRP7 promoter. Overall, there was a much reduced number of XL sites in the controls compared to the AtGRP7-GFP plants, and the XL sites did not map frequently to the very same positions in the independent replicates (Additional file 1: Figure S3). The GFP protein has recently also been found to be a suitable negative control in mammalian iCLIP studies $[62,63]$. Among the transcripts appearing in the controls were several tRNAs. Furthermore, transcripts encoding the small subunit of RIBULOSE BISPHOSPHATE CARBOXYLASE, one of the most abundant plant proteins, appeared in the controls at time point LL24. As more studies become available for Arabidopsis RBPs, the experience with frequently observed background XL sites will increase [64].

\section{iCLIP and RIP-seq identify an overlapping but not identi- cal set of target transcripts}

Global RIP-seq confirmed 53\% of the iCLIP targets at LL36 and 43\% at LL24, yielding a set of high-confidence binders. This is in the same range as observed for mammalian hnRNPH1 in a comparison of iCLIP and RIP which was performed without formaldehyde crosslinking [31]. RIP found 32\% of iCLIP targets of hnRNPH1, and in turn, $28 \%$ of the hnRNPH1 RIP targets were identified by iCLIP [31]. The identification of several hundred 
iCLIP targets indicates that AtGRP7 binds to a wide range of transcripts. Accordingly, AtGRP7 is one of the few proteins discovered in all three studies identifying mRNA interacting proteins globally in cell cultures, protoplasts, leaves, or etiolated seedlings [65].

Besides the high-confidence binders identified by iCLIP and confirmed by RIP-seq, we identified a suite of transcripts either by iCLIP only or RIP-seq only and validated a selection by RIP-qPCR. The identification of overlapping but not identical sets of targets indicates that both methods are complementary and generally enhance the identification of RBP targets. Crosslinking by formaldehyde and by UV light both have distinct advantages and drawbacks. UV does not crosslink proteins, thus limiting the analysis to RNAs immediately bound by the RBPs, whereas formaldehyde fixation yields direct and indirect targets of RBPs. In turn, formaldehyde has a higher crosslinking efficiency and thus may identify binding targets of lower abundance [32].

To identify RIP-seq targets we filtered for transcripts enriched in the precipitate over poly(A) RNA rather than enrichment relative to transcripts co-precipitating with GFP only or in a mock precipitation with RFP Trap beads. Our rationale was that the amount of RNAs coprecipitating with GFP alone was below the detection limit for photometric and fluorometric quantification. Therefore, we expected that precipitation of GFP-only plants or mock precipitation of AtGRP7-GFP with RFP Trap beads would produce low complexity libraries and consequently have a low coverage [66]. This is in line with our previous RIP-qPCR experiments and our validated data, showing low background of unspecific binding to mock controls (IP-) or GFP only. Similar results were obtained after mock precipitation with RFP Trap beads (IP-).

So far, two genome-wide investigations on RBP targets have been reported in Arabidopsis. RIP-seq on formaldehyde-treated plants expressing the serine/arginine-rich (SR)-like RBP SR45 fused to GFP identified 4262 SR45-assosciated RNAs, designated SARs, that were enriched upon precipitation with GFP antibodies from the SR-GFP plants over wild-type plants based on three biological replicates [5]. Of the SAR genes, 116 were differentially expressed in the sr45-1 mutant relative to the SR45.1-GFP line. A CLIP study of HLP1, an hnRNP A/B-like protein, identified 9031 binding sites from the sense transcripts of 5569 genes in one biological replicate [67]. Binding sites showed overrepresented A-rich and U-rich motifs predominantly near the poly(A) sites. Mutation of HLP1 causes altered polyadenylation in 429 of the 5569 target transcripts, including the flowering time gene $F C A$. Of interest, HLP1 binds also to the AtGRP7 5' UTR, although the consequences are not yet known [67].

\section{Binding mode of AtGRP7}

Because the iCLIP reads end at the XL site, insights can be obtained into motifs recognized by the RBPs with high resolution. AtGRP7 binds to all transcript regions with a preference for the $3^{\prime}$ UTR. The lowest number of $\mathrm{XL}$ sites mapped to the intron. This may also relate to the fact that introns are less represented in whole cell lysates as used for iCLIP here. The presence of significant $\mathrm{XL}$ sites in different regions of the transcripts points to different functions AtGRP7 may fulfill when binding to different regions. The U/C-rich motifs identified in the 5' UTR and exon by MEME were closely related, whereas they shared low similarities with the motif identified in the 3' UTR. This may point to different binding modes of AtGRP7 for different regions of the transcript. Differences in target site recognition in the 3' UTR versus other regions of its target transcripts have been observed for Musashi1 (MSI1) in human cell culture [68].

Notably, the UCUUCUUC motif located in the 5' UTR and the exon shows high similarity to two C/U-rich motifs enriched in targets of SR45 that are preferentially found in introns and 5' UTRs of the SAR transcripts compared to the non-SAR transcripts [5]. A comparison of the SAR genes to the AtGRP7 targets revealed that 150 of the 858 iCLIP targets and 61 of the high-confidence binders identified by both iCLIP and RIP-seq corresponded to SAR transcripts. Of the RIP-only targets, 503 were also found for SR45. This common set of targets may hint at overlapping or opposing functions of both proteins.

Previously, electrophoretic mobility shift assays revealed binding of AtGRP7 to the 3' UTR of its own pre-mRNA. Extensive deletion analysis and mutagenesis of the binding site unraveled a minimal sequence in the 3' UTR, UUC UGG [39]. This motif was subsequently employed to study the RNA-binding dynamics of NtGR-RBP1, a tobacco orthologue of $A t$ GRP7, by NMR [69]. Notably, iCLIP identified significant XL sites within a few nucleotides upstream and downstream of this motif, and an enriched pentamer maps to the motif, confirming the in vitro binding data in vivo (Additional file 1: Figure S7a). Furthermore, a binding site within the second half of the intron has been characterized in vitro $[14,18,40]$. Although this region did not return XL sites in the iCLIP studies, an intron motif was detected next to the determined in vitro binding site [40]. It has been observed that in vitro binding studies and in vivo CLIP experiments result in common but also distinct binding sites. For example, for the well-studied C. elegans PUF (Pumilio/FBF) protein, iCLIP peaks without the canonical binding element have been identified [33]. It should be noted that, in addition to the sequence context, secondary structure features of the RNA are relevant for binding which are not taken into account in vitro [70]. Furthermore, UV light irradiation in CLIP techniques leads to crosslinking of RNA and protein 
mainly at uridines and thus some binding sites may be less efficiently identified [71, 72].

The MEME motifs we identified are similar in length to motifs identified for other proteins with a single RRM, e.g. Arabidopsis SR45 [5] or hnRNPC1/C2 in HeLa cells [41]. It remains to be determined whether additional proteins that act synergistically or antagonistically with AtGRP7 may interact with the motifs. Furthermore, the accessory role the glycine-rich stretch has in RNA binding in addition to the RRM suggests that the binding site may be more extended [40].

\section{Impact of AtGRP7 on targets}

To unravel how AtGRP7 affects its targets at the RNA level, RNA-seq was performed on plants lacking AtGRP7 or having an elevated AtGRP7 level. An advantage of including the AtGRP7-ox plants is that, in the case of redundancies, changes in the mutant may be masked by other factors, yet upon overexpression a change may be seen. In the grp7-1 $8 i$ mutant, $4.46 \%$ of all DEGs at LL36 (93 of 2087) and 3.28\% of all DEGs at LL24 (24 of 731) are iCLIP targets (Additional file 1: Figures S8 and S11); $2.78 \%$ of all DEGs at LL36 (58 of 2,087) and 1.92\% of all DEGs at LL24 (14 of 731) are high-confidence targets also identified by RIP-seq. This compares well with the findings for the SR-like protein SR45, where 116 out of 4262 SR45 targets identified by RIP-seq (2.72\%) are differentially expressed in sr45-1 [5].

Importantly, our data suggest that the effect of AtGRP7 on its direct target genes is predominantly repressive (Additional file 1: Figure S12). RNA-seq revealed a similar number of transcripts upregulated or downregulated in AtGRP7-ox or grp7-1 $8 i$ plants compared to wt. In stark contrast, the bound targets are enriched for genes expressed at reduced levels in AtGRP7ox plants and upregulated in the grp $7-18 i$ mutant. This is seen for the high-confidence binders as well as for iCLIP and RIP-seq targets at both time points.

Among the high confidence binders were a number of transcripts implicated in stress responses. For example, $F A D 2$, which is required for salt tolerance, is downregulated in the AtGRP7-ox plants. It was reported that elevated levels of AtGRP7 have a negative effect on germination and seedling growth under salt stress conditions [20]. Furthermore, several cold-responsive transcripts were among the binding targets. AtGRP7 has been shown to promote freezing tolerance [20]. However, no noticeable difference in the expression of several transcripts implicated in freezing tolerance in either the mutant or AtGRP7-overexpressing plants were found in response to low temperature [20]. It should be noted that numerous cold-responsive transcripts are controlled by the circadian clock and thus upon exposure to low temperature an acute temperature response is overlaid by changes in the circadian oscillator at low temperature $[73,74]$. Therefore, more systematic investigations are required to monitor transcriptome changes in plants with altered AtGRP7 levels across a wider range of low temperatures. It remains possible that the role of AtGRP7 in freezing tolerance and its impact on the coldresponsive targets occur by another regulatory mechanism not seen at the transcript level. Furthermore, a number of transcripts bound by $A t$ GRP7 are associated with pathogen defense, including HSPRO2 and several WRKY transcription factors. Previously, we observed that AtGRP7 binds to the PDF1.2 transcript associated with jasmonic acid/ethylene-dependent defense against necrotrophic pathogens and negatively affects its expression, whereas it does not bind to the PR1 transcript and regulates PR1 transcription indirectly [36]. Thus, the determination of the AtGRP7 RNome and the dynamics of posttranscriptional networks controlled by AtGRP7 in response to pathogens will be revealing.

Although at first sight it seems counterintuitive that a relatively low proportion of the binding targets are differentially regulated at the RNA level, this has been observed before for in vivo binding substrates of both RBPs and transcription factors. A comprehensive iCLIP analysis of the SR protein family in mouse P19 cells revealed that each of the SRSF1 to SRSF7 factors crosslinks to thousands of target transcripts [62]. However, few transcripts are altered in the cytoplasm when individual SRSFs are knocked down, pointing to redundant functions in mRNA export. No strict correlation between binding of Saccharomyces cerevisiae Puf3p and altered abundance in puf3 deletion strains was observed, suggesting that other proteins may contribute to the regulation of the binding targets [75]. Comparison of the 2289 candidate ChIP target genes of the MADS-domain transcription factor APETALA1 (AP1), a key regulator of Arabidopsis flower development, with microarray data of the ap1 mutant revealed 249 genes with $>1.8$-fold differential expression [76]. Genome-wide identification of binding sites for two repressors of floral transition, FLOWERING LOCUS $\mathrm{C}$ and SHORT VEGETATIVE PHASE, revealed that 15 to $25 \%$ of the transcript changes were caused by direct regulation [77]. Furthermore, it should be kept in mind that crosslinking also captures transient interactions and thus may include interactions which are biologically "not meaningful" in the context investigated [78]. This does not rule out, however, that such an interaction may have functional consequences on target RNAs under other circumstances.

\section{Impact of AtGRP7 on circadian transcript oscillations}

The circadian clock controls transcription of about $30 \%$ of the Arabidopsis genome [79]. However, transcriptional rhythms lead to oscillations in mRNA steady-state abundance only if an mRNA is sufficiently short-lived [80]. Accordingly, increasing evidence points to regulation at 
the posttranscriptional level making an important contribution to the circadian transcriptome [81]. So far, it is known that AtGRP7 negatively autoregulates the oscillations of its own transcript and of the paralog AtGRP8 through binding to the pre-mRNAs. This causes alternative splicing and subsequent NMD. Mathematical modeling showed that this posttranscriptional regulation in response to rising AtGRP7 protein levels during the day indeed contributes to the sharp evening peaks of the AtGRP7 and AtGRP8 oscillations [82]. Here, we showed that elevated levels of AtGRP7 dampen circadian oscillations of two of its binding targets, DRM2 and CCL. Furthermore, AILP1, previously found to be negatively regulated by $A t \mathrm{GRP7}$ at the time of its circadian peak, was also a direct in vivo target [18]. Further studies will have to show how AtGRP7 controls the target genes identified here and how it may exert phase-specific effects that may result from the timing of its binding.

\section{Effect of AtGRP7 on RNA processing steps}

RNA-seq revealed alternative splicing events significantly changed in the grp7-1 $8 i$ loss-of-function mutant or AtGRP7-ox plants. This expands our previous data using a high-resolution RT-PCR based alternative splicing panel [16]. Several of the transcripts that showed changes in alternative splicing upon altered AtGRP7 levels were identified by iCLIP, RIP-seq, or both. For instance, of the highconfidence binders identified by both approaches, 40 showed changes in alternative splicing in grp7-1 $8 i$ and 71 in the AtGRP7-ox plants (Additional file 2: Table S16). A comparison of the splicing patterns of a suite of AtGRP7 targets at LL24 and LL36 did not reveal a prominent circadian clock-regulated influence of AtGRP7 on the splicing events.

The overall low number of splicing targets in our analysis may be due to the use of total cell extracts used for the immunoprecipitation, with nuclear RNA thus being less represented. For the genuine Arabidopsis splicing factor SR45, 331 of the 4316 RIP targets (7.7\%) were differentially spliced in the sr45-1 mutant [5]. A similar effect was seen for the RBP Musashi1 (MSI1), which is involved in glioblastoma multiforme, the most malignant form of brain cancer. Uren and coworkers identified a large number of binding sites in introns. However, only 26 genes with significant changes in exon usage upon MSI1 knockdown were found, pointing to a rather limited effect of MSI1 on alternative splicing, at least in the cell type studied [68]. In other cases more prevalent effects of an RBP on targets identified by CLIP techniques were found; e.g., $60 \%$ of transcripts that are aberrantly spliced upon knockdown of RBM10 are direct targets [83].

Binding targets that are not regulated at the level of steady-state abundance or alternative splicing may be subject to regulation at others steps, e.g., translation or
RNA transport. RNA-seq would fail to detect such effects. AtGRP7 has already been shown to participate in different steps of posttranscriptional control in the cell. This is supported by our finding that AtGRP7 binds transcripts with introns and without introns. Here we find evidence that AtGRP7 may also regulate alternative polyadenylation of selected target transcripts. Furthermore, a role for RBPs in translational regulation in the circadian system is proposed by a recent study showing proteins with rhythmic steady-state abundance despite a constant mRNA level [84].

AtGRP7 has also been shown to function as an RNA chaperone [17]. In line with this, we find transcripts with clusters of crosslink sites, pointing to cooperative binding (Additional file 1: Figure S4b, o). In-depth studies are required to mechanistically connect AtGRP7 binding to different transcript regions with function.

\section{Conclusions}

Adaptation of iCLIP previously used for UV-irradiated mammalian cell monolayers [10] or C. elegans to plant tissue significantly expands the toolkit to identify RNAprotein interactions in planta. iCLIP combined with RIP-seq identified a set of 452 high-confidence targets of AtGRP7, some of which are regulated by AtGRP7 at the level of steady-state abundance or splicing. Targeted manipulation of the binding motifs detected in the vicinity of the XL sites will reveal their contribution to the control of the targets by AtGRP7 in the plant. The binding motifs discovered here could now be used to predict additional AtGRP7 targets. As numerous stressresponsive transcripts were found among the highconfidence binders, a next step is to unravel how posttranscriptional networks controlled by AtGRP7 are reconfigured under stress.

\section{Methods \\ Plant material}

The line AtGRP7::AtGRP7-GFP expresses an AtGRP7GFP fusion under control of $1.4 \mathrm{~kb}$ of the AtGRP7 promoter and the AtGRP7 5' UTR, intron, and 3' UTR in the grp7-1 T-DNA mutant, and the line AtGRP7::GFP expresses GFP only under control of $1.4 \mathrm{~kb}$ of the AtGRP7 promoter, the AtGRP7 5' UTR, and 3' UTR $[16,85]$. The line AtGRP7::AtGRP7 $R^{49} \mathrm{Q}-G F P$ expresses an RNA-binding mutant version with $\mathrm{Arg}^{49}$ exchanged for Gln [16]. The grp7-1 $8 i$ line has an RNAi construct against AtGRP8 to counteract elevated AtGRP8 levels due to relief of repression by AtGRP7 in grp7-1 [21]. AtGRP7-ox plants express the AtGRP7 coding sequence under control of the cauliflower mosaic virus (CaMV) $35 \mathrm{~S}$ promoter [86]. 


\section{Plant growth}

Arabidopsis seeds were surface-sterilized and sown on half-strength MS (Murashige-Skoog; Duchefa) plates

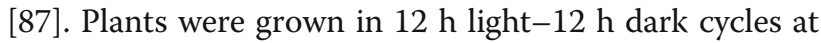
$20{ }^{\circ} \mathrm{C}$ in Percival incubators (CLF laboratories) followed by free run under continuous light (LL). For RNA analysis, aerial tissue was harvested at subjective dawn or dusk for iCLIP, RIP, and RNA-seq or at 2-h intervals starting $4 \mathrm{~h}$ before subjective dawn for qPCR. At least ten plants were bulked for each sample per replicate.

\section{iCLIP}

The iCLIP procedure developed by König and co-workers was adapted to plant tissue [55]. Seedlings on plates were subjected to irradiation with 254-nm UV light at a dose of $500 \mathrm{~mJ} / \mathrm{cm}^{2}$ in a UVP CL-1000 UV crosslinker on ice. The plant material was quick-frozen in liquid $\mathrm{N}_{2}$ and ground to a homogeneous powder with mortar and pestle. Cell lysis buffer $(50 \mathrm{mM}$ Tris- $\mathrm{HCl}, \mathrm{pH} 7.5,150 \mathrm{mM} \mathrm{NaCl}$, $4 \mathrm{mM} \mathrm{MgCl}, 0.25 \%$ Igepal CA-630, 1\% SDS, $0.25 \%$ sodium deoxycholate, $5 \mathrm{mM}$ DTT, Complete Protease Inhibitor (Roche), $100 \mathrm{U} / \mathrm{mL}$ RiboLock (Thermo Fisher), $1 \mathrm{mM}$ phenylmethylsulfonylfluorid) was added to the powder. Lysates were precleared with sepharose beads for $1 \mathrm{~h}$ at $4{ }^{\circ} \mathrm{C}$ with constant rotation and subjected to immunoprecipitation with GFP Trap beads (Chromotek) or mock precipitation with RFP Trap beads (Chromotek). The RNA-protein complexes were precipitated for $1 \mathrm{~h}$ at $4{ }^{\circ} \mathrm{C}$ with constant rotation. The beads were washed four times with $1 \mathrm{~mL}$ cooled RIP-washing buffer $(50 \mathrm{mM}$ Tris$\mathrm{HCl}, \mathrm{pH} 7.5,500 \mathrm{mM} \mathrm{NaCl}, 4 \mathrm{mM} \mathrm{MgCl}_{2}, 0.5 \%$ Igepal CA-630, 1\% SDS, $0.5 \%$ sodium deoxycholate, sodium salt, $2 \mathrm{M}$ urea, $2 \mathrm{mM}$ DTT, Complete Protease Inhibitor) and washed twice with $1 \mathrm{ml}$ cooled original iCLIP wash butter (20 mM Tris- $\mathrm{HCl} \mathrm{pH} \mathrm{7.4,} 10 \mathrm{mM} \mathrm{MgCl}_{2}, 0.2 \%$ Tween 20 ) [10]. On the beads, the precipitate was treated with $2 \mu \mathrm{l}$ Turbo DNase for $10 \mathrm{~min}$ at $37^{\circ} \mathrm{C}$ (Thermo Fisher). For RNase digestion, 6.7 U RNase I (Thermo Fisher) were added. For library preparation, the RNAs were dephosphorylated and the L3 linker (Additional file 2: Table S18) was ligated to the 3 ' ends using RNA ligase (NEB).

The $5^{\prime}$ termini were labeled using $\left[\gamma-{ }^{32} \mathrm{P}\right]$ ATP and polynucleotide kinase and the covalently linked RNAprotein complexes were separated on a $4-12 \%$ NuPAGE Bis-Tris gel (Thermo Scientific), and electroblotted onto a nitrocellulose membrane. Upon autoradiography, the regions above the fusion protein were cut out and subjected to proteinase $\mathrm{K}$ treatment, leaving a polypeptide at the interaction site. Subsequently, RNA was isolated from the membrane using TriReagent and reverse transcribed using primers containing a cleavable adapter region and individual barcode sequences (Additional file 2: Table S18).
After $\mathrm{NaOH}$ treatment, the cDNA was purified on a $6 \%$ urea-polyacrylamide gel and fragments in the size range of approximately 70-85 nucleotides (high, H), 85-120 nucleotides (medium, M), and 120-200 nucleotides (low, L), respectively, were eluted from the gel (Additional file 1: Figure S2c). The cDNAs were then circularized using CircLigase II (Epicentre) and an oligonucleotide (Cutoligo) was annealed to generate a BamHI restriction site. Relineariztion via BamHI digestion results in adapters at both ends of the cDNA which were then PCR-amplified. After PCR optimization the three size fractions $(\mathrm{H}, \mathrm{M}, \mathrm{L})$ were pooled with a ratio of $1: 1: 1$, concentrations were assessed with Qubit dsDNA HS Assay Kit (Thermo Scientific), and $10 \mathrm{nM}$ of the libraries were submitted to high-throughput sequencing after multiplexing of multiple samples. Sequencing was carried out using an Illumina HiSeq2500 (Eurofins) with 50-nucleotide single-end reads or at the Genomics Center of the Max-Planck-Institute for Developmental Biology, Tuebingen, with 100-nucleotide single-end reads.

\section{RIP-seq}

Plants grown in $12 \mathrm{~h}$ light-12 h dark cycles for 16 days and subsequently shifted to continuous light were vacuum-infiltrated with $1 \%$ formaldehyde for $15 \mathrm{~min}$ at LL36 or LL24, followed by quenching with $125 \mathrm{mM}$ glycine. A whole-cell extract was prepared in RIP-lysis buffer $(50 \mathrm{mM}$ Tris- $\mathrm{HCl} \mathrm{pH} 7.5,150 \mathrm{mM} \mathrm{NaCl}, 4 \mathrm{mM}$ $\mathrm{MgCl}_{2}, 0.25 \%$ Igepal CA-630, $1 \%$ SDS, $5 \mathrm{mM}$ DTT, $10 \mathrm{mM}$ vanadylribonucleosid complex, $100 \mathrm{U} / \mathrm{ml}$ RiboLock (Thermo Fisher), $1 \mathrm{mM}$ phenylmethylsulfonylfluorid, and Complete Protease Inhibitor). The extract was pre-cleared with Sepharose beads and subjected to immunoprecipitation with GFP-Trap beads (Chromotek), hereafter called IP+. After extensive washing with RIP washing buffer (50 mM Tris- $\mathrm{HCl} \mathrm{pH} 7.5,500 \mathrm{mM} \mathrm{NaCl}, 4 \mathrm{mM} \mathrm{MgCl}_{2}$, $5 \mathrm{mM}$ DTT, $0.5 \%$ Igepal CA-630, 1\% SDS, 0.5\% sodium deoxycholate, $2 \mathrm{M}$ urea), co-precipitated RNAs were eluted with TriReagent and treated with DNase (Promega).

Libraries were prepared from three biological replicates using the Illumina TrueSeq Sample preparation kit, except for omitting the two rounds of poly(A) selection commonly used for total RNA as a starting material. Sequencing was carried out using an Illumina HiSeq2000 at the Genomics Center of the Max-Planck-Institute for Developmental Biology, Tuebingen, with 100-nucleotide single-end reads.

\section{RNA-seq}

Col-2 wt plants, AtGRP7-ox plants, and the grp7-1 $8 i$ line were harvested at LL36 or LL24 in parallel to the RIP-seq samples. Total RNA was isolated using the Universal RNA purification Kit (including DNase digestion; EURx, Roboklon). Total RNA integrity was analyzed on 
an Agilent 2100 Bioanalyzer using the Agilent RNA 6000 Nano Kit and RNA showing a RIN > 8 were further processed. Libraries were prepared from three biological replicates using the TruSeq RNA sample prep kit v2. Sequencing was carried out using an Illumina HiSeq2000 at the Genomics Center of the Max-Planck-Institute for Developmental Biology, Tuebingen, with 100-nucleotide paired-end reads.

\section{Bioinformatics \\ iCLIP-seq}

Raw iCLIP reads were subjected to 3 ' adapter trimming and quality filtering using cutadapt version 1.9.1 (https:// github.com/marcelm/cutadapt). Only reads with a minimal length of 15 nucleotides and a quality score of 20 were kept. The trimmed and filtered reads were de-multiplexed by an in house python script available at https:/github.com/GrosseLab/iCLIP. Identical reads including the same random barcode sequence were considered PCR duplicates and hence removed. The barcodes were trimmed from the remaining reads using barcodeRemoval from PIPE-CLIP [88]. The resulting reads were mapped to the $A$. thaliana TAIR10 reference genome with STAR v2.5.2a [89] using the additional transcript annotation file atRTD.gff from atRTD, a novel reference transcriptome containing more than 32,500 additional transcript isoforms [30]. Up to three mismatches were allowed, and only reads mapping uniquely were kept.

Putative XL sites were determined as described previously with minor modifications [10]. XL sites were determined separately for each transcript region due to possible differences in their expression level. For this, introns, exons (concatenated), 5' UTR, and 3' UTR were defined as separate regions based on the representative gene model deposited in TAIR10. For the following steps, only the XL sites ( -1 position of the reads) were considered, whereas the rest of the reads were omitted.

To determine the significance of each XL site, the FDR was determined. For this, each XL site was extended by 15 nucleotides in both directions and the number of crosslinks were added up for each position, defining a height $h$. A distribution of the occurrence of each height in the corresponding region was specified, $\left\{n_{1}, n_{2}, \ldots n_{h}, \ldots n_{H-1}, n_{H}\right\}$, where $H$ is the maximal height appearing in the region and $n_{h}$ expresses the number of XL sites with height $h$. Therefore, the probability of an observed height of at least $h$ can be expressed by:

$$
P(h)=\frac{\sum_{i=h}^{H} n_{i}}{N}
$$

To discriminate the observed heights from randomly appearing crosslinks, a randomized height distribution was generated 100 times for each region. For this, the same number of $N$ crosslinks were distributed uniformly in the corresponding regions, assembling a background. The mean and standard deviation of each randomly generated height $\left(\mu_{h}, \sigma_{h}\right)$ were used to compute the FDR for every observed height:

$$
F D R(h)=\frac{\left(\mu_{h}+, \sigma_{h}\right)}{P(h)}
$$

Crosslink positions with a FDR $<0.05$ were considered as significant.

To increase reliability, especially in large regions with few crosslinks, the whole significance procedure (assigning XL sites to FDR) was repeated 1000 times to accommodate for variability. Crosslink sites with a FDR $<0.05$ in at least $95 \%$ of the re-runs were further examined. Only those crosslinks that occurred at the same position in all but one of the replicates were considered for downstream analyses. In datasets with only two replicates, the XLs occurring in both were considered. This computation was performed for the AtGRP7-GFP samples and the $A t \mathrm{GRP7} \mathrm{R}^{49} \mathrm{Q}-\mathrm{GFP}$ and GFP-only control samples separately. Transcripts with significant crosslink positions in the control samples were removed from the putative AtGRP7 targets.

\section{Motif discovery}

A motif search was performed individually on each of the previously defined regions using the MEME suite 4.11.1 [38]. Each XL site was extended by ten nucleotides in either direction. The extraction of genomic sequences was done using bedtools [90], with the addition that thymine $(\mathrm{T})$ occurrences were replaced with uracil (U). The background model was determined by randomly assigning the exact amount of crosslinks uniformly in every region of the transcripts where significant crosslink sites have been identified.

\section{Determination of pentamer enrichment at XL sites}

The Z-score analysis was performed separately for the different transcript regions (introns, exons, 5' UTRs, and 3' UTRs). Crosslinks on transcripts antisense to the transcriptional direction of the corresponding gene were removed before proceeding. A random background was generated 100 times for each region, assigning random crosslinks uniformly across the corresponding region. All XL sites (experimental and random) were extended by ten nucleotides in either direction, resulting in 21nucleotide-long genomic sequences. The pentamer frequency was calculated for each region independently. The Z-score for every pentamer in every region was then determined as follows: 


$$
\operatorname{Zscore}(P)=\frac{f_{P_{\text {exp }}-\mu f_{P_{\text {control }}}}}{\sigma f_{P_{\text {control }}}}
$$

where $P$ is pentamer, $f_{\text {Pexp }}$ is the frequency of the observed pentamer, $\mu f_{\text {Pcontrol }}$ is the mean frequency of the pentamer in the control dataset, and $\sigma f_{\text {Pcontrol }}$ is the standard deviation of the frequency in the control dataset for the given pentamer.

\section{RIP-seq}

The evaluation of RIP-seq data was done similarly to [34]. Raw RIP-seq reads were subjected to quality trimming and filtering by Sickle 11.2 (https://github.com/najoshi/sickle) using parameters -1 50 -q 20. The trimmed and filtered reads were mapped to the A. thaliana transcriptome defined by atRTD.gff using STAR v2.5.2a [89] with the parameter -quantMode TranscriptomeSAM. Estimated read counts per transcript were obtained by Salmon v0.8.2 [91] and summarized into estimated read counts per gene by tximport [92]. rRNA, mitochondrial, and chloroplast genes were excluded from the analysis. Transcripts enriched in the RIP samples relative to the RNA-seq samples were detected by edgeR as described in the tximport vignette (https:// github.com/mikelove/tximport/blob/master/vignettes/ tximport.md). Genes with a FDR $<0.001$ and a $\log _{2}$-fold change $\geq 0.5$ were considered putative RIP targets.

\section{RNA-seq}

For the analysis of the RNA-seq data, only the 100-bp R1 reads coming from the original paired-end reads were used for further analysis. Raw RNA-seq reads were processed by the same pipeline as raw RIP-seq reads (see above) with the only exception of Sickle parameter -q 30 . DEGs between AtGRP7-ox and wt as well as between grp7-1 $8 i$ and wt were detected by edgeR (see above). Genes with a FDR $<0.05$ and a TPM $>1$ in at least one of the genotypes were considered putative DEGs.

\section{Changes in alternative splicing events}

The estimated read counts per transcript obtained from Salmon were processed by tximport [92]. Alternative splicing events in the atRTD annotation [30] were classified using SUPPA [91]. Transcripts having just one annotated isoform were excluded from the analysis. Percentage spliced in (PSI) values, defined as the ratio between the TPM of the isoform including the event and the overall TPM of all isoforms for each splicing event for all genotypes, in wt and AtGRP7-ox as well as wt and grp 7-1 $8 i$ were compared and tested for significance. Events with a corrected $p$ value $<0.01$ and $|\Delta \mathrm{PSI}|>0.1$ were considered significant.

\section{RT-PCR and real-time PCR}

For semiquantitative RT-PCR, retrotranscribed RNA was amplified with Taq polymerase in a total volume of $20 \mu$ l. To determine the linear range of amplification for each primer pair, samples were withdrawn after 24, 26, $28,30,32$, and 34 cycles. PCR products were separated on agarose gels and visualized by ethidium-bromide staining. qPCR was performed in a volume of $10 \mu \mathrm{l}$ with the iTaq SYBR GREEN supermix (Biorad) using 45 cycles of $15 \mathrm{~s}$ at $95{ }^{\circ} \mathrm{C}$ and $30 \mathrm{~s}$ at $60{ }^{\circ} \mathrm{C}$ for RIP-qPCR and the circadian RNA kinetics, or in a volume of $20 \mu \mathrm{l}$ with EvaGreen and GoTaq polymerase (Promega) using 45 cycles of $15 \mathrm{~s}$ at $95{ }^{\circ} \mathrm{C}, 20 \mathrm{~s}$ at $55^{\circ} \mathrm{C}$, and $20 \mathrm{~s}$ at $72{ }^{\circ} \mathrm{C}$ to investigate differentially expressed genes, respectively, in a CFX96 cycler (Biorad). $\mathrm{C}_{\mathrm{q}}$ values were determined and relative expression levels were calculated based on non-equal efficiencies for each primer pair [93]. Data were normalized to PP2A (At1g13320) and expressed as the mean expression levels of the independent biological replicates with two technical replicates each \pm standard deviation or as indicated in the figure legend. RIP-qPCR was performed as described [16]. Primers are listed in Additional file 2: Table S18.

\section{Immunoblot analysis}

Immunoblot analysis of lysates and chemiluminescence detection was done as described [94]. Primary antibodies were the antipeptide antibody against AtGRP7 (rabbit; dilution 1:2500), which discriminates AtGRP7 from AtGRP8 and lacks a signal in grp7-1 [21], a polyclonal serum against LHCP (rabbit; 1:25,000) [95], and a monoclonal antibody against GFP (Roche catalog number 11 814460 001; mouse; dilution 1:1000). Secondary antibodies were HRP-coupled anti-rabbit IgG (Sigma-Aldrich catalog number A 0545; dilution 1:5000) or HRP-coupled anti-mouse IgG (Sigma-Aldrich catalog number A0168; dilution 1:2500).

\section{Additional files}

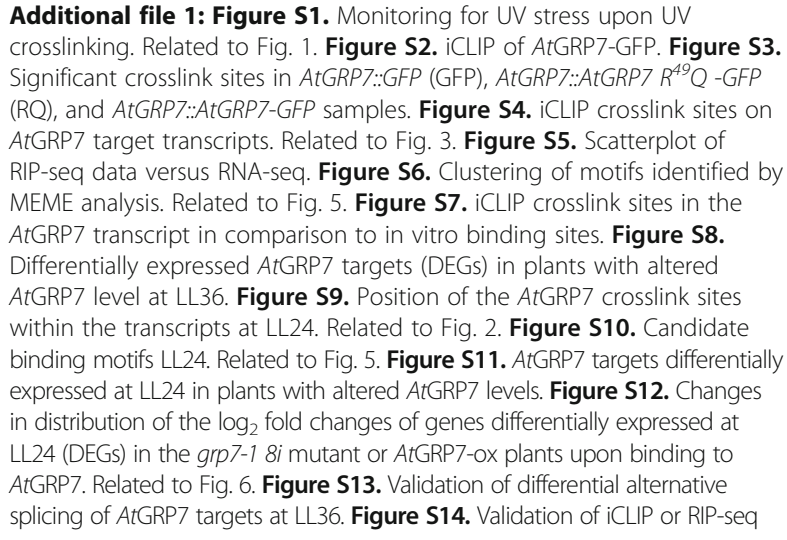


alternative splicing targets by RIP RT-PCR. Figure S15 Validation of differential alternative splicing of AtGRP7 targets at LL24. (PDF $4700 \mathrm{~kb}$ )

Additional file 2: Table S1. Preprocessing of the iCLIP sequencing libraries at LL36 and mapping statistics. Table S2. AtGRP7 iCLIP targets at LL36. Table S3. RIP read statistics at LL36. Table S4. RIP-seq targets at LL36. Table S5. High-confidence binders identified by both iCLIP and RIP-seq at LL36. Table S6. Pentamers enriched in the vicinity of the crosslink sites at LL36. Table S7. RNA-seq read statistics at LL36. Table S8. Differentially expressed AtGRP7 targets at LL36. Table S9. Preprocessing of the iCLIP sequencing libraries at LL24 and mapping statistics. Table S10. AtGRP7 iCLIP targets at LL24. Table S11. RIP read statistics at LL24. Table S12. RIP-seq targets at LL24. Table S13. High-confidence binders identified by both iCLIP and RIP-seq at LL24. Table S14. RNA-seq read statistics at LL24. Table S15. Differentially expressed AtGRP7 targets at LL24. Table S16. Changes in alternative splicing among AtGRP7 targets. Table S17. Alternative splicing of AtGRP7 targets at LL24 vs. LL36. Table S18. Primers used in this study. (XLSX 888 kb)

\section{Abbreviations}

CLIP: Crosslinking immunoprecipitation; DEG: differentially expressed gene; FDR: False discovery rate; GFP: GREEN FLUORESCENT PROTEIN;

iCLIP: Individual nucleotide resolution cross-linking and immunoprecipitation; LL: Continuous light; NMD: Nonsense-mediated decay; PSI: Percent spliced in; PTC: Premature termination codon; RBP: RNA-binding protein; RFP: RED FLUORESCENT PROTEIN; RIP: RNA immunoprecipitation; RNA-seq: highthroughput sequencing of CDNAs; RRM: RNA recognition motif; TPM: Transcripts per million; UTR: Untranslated region; wt: Wild type; $\mathrm{XL}:$ Crosslink.

\section{Acknowledgements}

We thank K. Neudorf for expert technical assistance, K. Lamkiewicz for developing a tool for demultiplexing of iCLIP reads and preliminary iCLIP analyses, and Dr. J. König, Prof. Dr. M. Feldbrügge and L. Olgeiser for helpful advice on the iCLIP procedure. We acknowledge Dr. C. Lanz and Prof. Dr. D. Weigel for use of the sequencing facilities at the Genomics Center MaxPlanck-Institute for Developmental Biology, Tübingen. We thank Dr. J. Mateos and Dr. A. Steffen for critical comments on the manuscript.

\section{Funding}

This work was supported by the German Research Foundation through grants STA 653/6-1, GR 3526/3-1, and SPP1530. KM and CN were supported by a PhD fellowship of the German National Academic Foundation.

\section{Availability of data and materials}

All the data reported in this paper can be found in the Gene Expression Omnibus database with the accession numbers GSE99427 (iCLIP data) [96], GSE99615 (RNA-seq data) [97], GSE99464 (RIP-seq data) [98], and GSE99616 (SuperSeries) [99]. Information and requests for reagents: dorothee.staiger@uni-bielefeld.de.

\section{Authors' contributions}

$D S$, TK, and IG designed the project. KM, TK, and CN performed the experiments. CW and ML performed the bioinformatics analyses. DS wrote the manuscript. All authors read and approved the final manuscript.

\section{Ethics approval and consent to participate}

Not applicable.

\section{Competing interests}

The authors declare that they have no competing interests.

\section{Publisher's Note}

Springer Nature remains neutral with regard to jurisdictional claims in published maps and institutional affiliations.

\section{Author details}

'RNA Biology and Molecular Physiology, Faculty of Biology, Bielefeld University, Bielefeld, Germany. ${ }^{2}$ Institute of Computer Science, Martin Luther University Halle-Wittenberg, Halle, Germany. ${ }^{3}$ German Centre for Integrative Biodiversity Research (iDiv) Halle-Jena-Leipzig, Leipzig, Germany.
Received: 1 June 2017 Accepted: 29 September 2017

Published online: 31 October 2017

\section{References}

1. Silverman IM, Li F, Gregory BD. Genomic era analyses of RNA secondary structure and RNA-binding proteins reveal their significance to posttranscriptional regulation in plants. Plant Sci. 2013;205-206:55-62. doi:10. 1016/j.plantsci.2013.01.009.

2. Schmitz-Linneweber C, Williams-Carrier R, Barkan A. RNA immunoprecipitation and microarray analysis show a chloroplast Pentatricopeptide repeat protein to be associated with the $5^{\prime}$ region of mRNAs whose translation it activates. Plant Cell. 2005;17:2791-804. doi:10. 1105/tpc.105.034454.

3. Terzi LC, Simpson GG. Arabidopsis RNA immunoprecipitation. Plant J. 2009; 59:163-8. doi:10.1111/j.1365-313X.2009.03859.x.

4. Köster T, Staiger D. RNA-binding protein immunoprecipitation from whole-cell extracts. Methods Mol Biol. 2014;1062:679-95. doi:10.1007/978-1-62703-580-4_35.

5. Xing D, Wang Y, Hamilton M, Ben-Hur A, Reddy ASN. Transcriptome-wide identification of RNA targets of Arabidopsis SERINE/ARGININE-RICH45 uncovers the unexpected roles of this RNA binding protein in RNA processing. Plant Cell. 2015;27:3294-308. doi:10.1105/tpc.15.00641.

6. Ule J, Jensen KB, Ruggiu M, Mele A, Ule A, Darnell RB. CLIP identifies Novaregulated RNA networks in the brain. Science. 2003;302:1212-5. doi:10.1126/ science.1090095.

7. Urlaub H, Hartmuth K, Lührmann R. A two-tracked approach to analyze RNA-protein crosslinking sites in native, nonlabeled small nuclear ribonucleoprotein particles. Methods. 2002;26:170-81. doi:10.1016/S10462023(02)00020-8.

8. Haberman N, Huppertz I, Attig J, König J, Wang Z, Hauer C, Hentze MW Kulozik AE, Le Hir H, Curk T, et al. Insights into the design and interpretation of iCLIP experiments. Genome Biol. 2017;18:7. doi:10.1186/s13059-016-1130-x.

9. Sugimoto Y, Konig J, Hussain S, Zupan B, Curk T, Frye M, Ule J. Analysis of CLIP and iCLIP methods for nucleotide-resolution studies of protein-RNA interactions. Genome Biol. 2012;13:R67. doi:10.1186/gb-2012-13-8-r67.

10. König J, Zarnack K, Rot G, Curk T, Kayikci M, Zupan B, Turner DJ, Luscombe NM, Ule J. iCLIP reveals the function of hnRNP particles in splicing at individual nucleotide resolution. Nat Struct Mol Biol. 2010;17:909-15. doi:10. 1038/nsmb.1838.

11. Staiger D, Shin J, Johansson M, Davis SJ. The circadian clock goes genomic. Genome Biol. 2013;14:208. doi:10.1186/gb-2013-14-6-208.

12. Heintzen C, Melzer S, Fischer R, Kappeler S, Apel K, Staiger D. A light- and temperature-entrained circadian clock controls expression of transcripts encoding nuclear proteins with homology to RNA-binding proteins in meristematic tissue. Plant J. 1994;5:799-813.

13. Carpenter CD, Kreps JA, Simon AE. Genes encoding glycine-rich Arabidopsis thaliana proteins with RNA-binding motifs are influenced by cold treatment and an endogenous circadian rhythm. Plant Physiol. 1994;104:1015-25.

14. Staiger D, Zecca L, Wieczorek Kirk DA, Apel K, Eckstein L. The circadian clock regulated RNA-binding protein AtGRP7 autoregulates its expression by influencing alternative splicing of its own pre-mRNA. Plant J. 2003;33:361-71.

15. Schöning JC, Streitner C, Meyer IM, Gao Y, Staiger D. Reciprocal regulation of glycine-rich RNA-binding proteins via an interlocked feedback loop coupling alternative splicing to nonsense-mediated decay in Arabidopsis. Nucleic Acids Res. 2008;36:6977-87. doi:10.1093/nar/gkn847.

16. Streitner C, Köster T, Simpson CG, Shaw P, Danisman S, Brown JWS, Staiger D. An hnRNP-like RNA-binding protein affects alternative splicing by in vivo interaction with target transcripts in Arabidopsis thaliana. Nucleic Acids Res. 2012;40:11240-55. doi:10.1093/nar/gks873.

17. Kim JS, Park SJ, Kwak KJ, Kim YO, Kim JY, Song J, Jang B, Jung CH, Kang H. Cold shock domain proteins and glycine-rich RNA-binding proteins from Arabidopsis thaliana can promote the cold adaptation process in Escherichia coli. Nucleic Acids Res. 2007;35:506-16. doi:10.1093/nar/gkl1076.

18. Schöning JC, Streitner C, Page DR, Hennig S, Uchida K, Wolf E, Furuya M, Staiger D. Autoregulation of the circadian slave oscillator component AtGRP7 and regulation of its targets is impaired by a single RNA recognition motif point mutation. Plant J. 2007;52:1119-30. doi:10.1111/j.1365-313X. 2007.03302.x.

19. Jeong, B-r, Lin Y, Joe A, Guo M, Korneli C, Yang H, Wang P, Yu M, Cerny RL, Staiger D, et al. Structure function analysis of an ADP-ribosyltransferase type III effector and its RNA-binding target in plant immunity. J Biol Chem. 2011; 286:43272-81. doi:10.1074/jbc.M111.290122. 
20. Kim JS, Jung HJ, Lee HJ, Kim KA, Goh CH, Woo Y, Oh SH, Han YS, Kang $H$. Glycine-rich RNA-binding protein7 affects abiotic stress responses by regulating stomata opening and closing in Arabidopsis thaliana. Plant J. 2008;55:455-66. doi:10.1111/j.1365-313X.2008.03518.x.

21. Streitner C, Danisman S, Wehrle F, Schöning JC, Alfano JR, Staiger D. The small glycine-rich RNA-binding protein AtGRP7 promotes floral transition in Arabidopsis thaliana. Plant J. 2008;56:239-50. doi:10.1111/j.1365-313X.2008.03591.X.

22. Löhr B, Streitner C, Steffen A, Lange T, Staiger D. A glycine-rich RNA-binding protein affects gibberellin biosynthesis in Arabidopsis. Mol Biol Rep. 2014;41: 439-45. doi:10.1007/s11033-013-2878-7.

23. Rothbauer U, Zolghadr K, Muyldermans S, Schepers A, Cardoso MC, Leonhardt $\mathrm{H}$. A versatile nanotrap for biochemical and functional studies with fluorescent fusion proteins. Mol Cell Proteomics. 2008;7:282-9. doi:10. 1074/mcp.M700342-MCP200.

24. Prasad A, Porter DF, Kroll-Conner PL, Mohanty I, Ryan AR, Crittenden SL, Wickens M, Kimble J. The PUF binding landscape in metazoan germ cells. RNA. 2016;22:1026-43. doi:10.1261/rna.055871.116.

25. He R, Drury GE, Rotari VI, Gordon A, Willer M, Farzaneh T, Woltering EJ, Gallois P. Metacaspase-8 modulates programmed cell death induced by ultraviolet light and $\mathrm{H} 2 \mathrm{O} 2$ in Arabidopsis. J Biol Chem. 2008;283:774-83. doi: 10.1074/jbc.M704185200

26. Frohnmeyer $\mathrm{H}$, Staiger D. Ultraviolet-B radiation-mediated responses in plants. balancing damage and protection. Plant Physiol. 2003;133:1420-8. doi:10.1104/pp.103.030049.

27. Castells E, Molinier J, Drevensek S, Genschik P, Barneche F, Bowler C. det1-1induced UV-C hyposensitivity through UVR3 and PHR1 photolyase gene over-expression. Plant J. 2010;63:392-404. doi:10.1111/j.1365-313X.2010. 04249.x.

28. Wituszynska W, Szechynska-Hebda M, Sobczak M, Rusaczonek A, KozkowskaMakulska A, Witon D, Karpinski S. LESION SIMULATING DISEASE 1 and ENHANCED DISEASE SUSCEPTIBILITY 1 differentially regulate UV-C-induced photooxidative stress signalling and programmed cell death in Arabidopsis thaliana. Plant Cell Environ. 2015;38:315-30. doi:10.1111/pce.12288.

29. Köster T, Haas M, Staiger D. The RIPper Case: Identification of RNA-binding protein targets by RNA immunoprecipitation. Methods Mol Biol. 2014;1158: 107-21. doi:10.1007/978-1-4939-0700-7_7.

30. Zhang R, Calixto CPG, Tzioutziou NA, James AB, Simpson CG, Guo W, Marquez $Y$, Kalyna M, Patro R, Eyras E, et al. AtRTD-a comprehensive reference transcript dataset resource for accurate quantification of transcript-specific expression in Arabidopsis thaliana. New Phytol. 2015;208: 96-101. doi:10.1111/nph.13545.

31. Uren PJ, Bahrami-Samani E, de Araujo PR, Vogel C, Qiao M, Burns SC, Smith $A D$, Penalva LOF. High-throughput analyses of hnRNP H1 dissects its multifunctional aspect. RNA Biol. 2016;13:400-11. doi:10.1080/15476286.2015.1138030.

32. Fecko CJ, Munson KM, Saunders A, Sun G, Begley TP, Lis JT, Webb WW. Comparison of femtosecond laser and continuous wave UV sources for protein-nucleic acid crosslinking. Photochem Photobiol. 2007:83:1394-404. doi:10.1111/j.1751-1097.2007.00179.x.

33. Darnell RB. HITS-CLIP: panoramic views of protein-RNA regulation in living cells. Wiley Interdiscip Rev. 2010;1:266-86. doi:10.1002/wrna.31.

34. Bersani C, Huss M, Giacomello S, Xu L-D, Bianchi J, Eriksson S, Jerhammar F, Alexeyenko A, Vilborg A, Lundeberg J, et al. Genome-wide identification of Wig-1 mRNA targets by RIP-Seq analysis. Oncotarget. 2015;7:1895-911. doi: 10.18632/oncotarget.6557.

35. Hansen HT, Rasmussen SH, Adolph SK, Plass M, Krogh A, Sanford J, Nielsen FC, Christiansen J. Drosophila Imp iCLIP identifies an RNA assemblage coordinating F-actin formation. Genome Biol. 2015;16:123. doi:10.1186/ s13059-015-0687-0.

36. Hackmann C, Korneli C, Kutyniok M, Köster T, Wiedenlübbert M, Müller C, Staiger D. Salicylic acid-dependent and -independent impact of an RNA-binding protein on plant immunity. Plant Cell Environ. 2014;37:696-706. doi:10.1111/pce.12188.

37. Ståhlberg A, Håkansson J, Xian X, Semb H, Kubista M. Properties of the reverse transcription reaction in mRNA quantification. Clin Chem. 2004;50: 509-15. doi:10.1373/clinchem.2003.026161.

38. Bailey TL, Boden M, Buske FA, Frith M, Grant CE, Clementi L, Ren J, Li WW, Noble WS. MEME Suite: tools for motif discovery and searching. Nucleic Acids Res. 2009;37:W202-8. doi:10.1093/nar/gkp335.

39. Schüttpelz M, Schöning JC, Doose S, Neuweiler H, Peters E, Staiger D, Sauer M. Changes of conformational dynamics of mRNA upon AtGRP7 binding studied by fluorescence correlation spectroscopy. J Am Chem Soc. 2008; 130:9507-13. doi:10.1021/ja801994z.
40. Leder V, Lummer M, Tegeler K, Humpert F, Lewinski M, Schüttpelz M, Staiger D. Mutational definition of binding requirements of an hnRNP-like protein in Arabidopsis using fluorescence correlation spectroscopy. Biochem Biophys Res Commun. 2014;453:69-74. doi:10.1016/j.bbrc.2014.09.056.

41. Zarnack K, König J, Tajnik M, Martincorena I, Eustermann S, Stévant I, Reyes A, Anders S, Luscombe Nicholas M, Ule J. Direct Competition between hnRNP C and U2AF65 protects the transcriptome from the exonization of Alu elements. Cell. 2013;152:453-66. doi:10.1016/j.cell.2012.12.023.

42. Wickramasinghe VO, Gonzàlez-Porta M, Perera D, Bartolozzi AR, Sibley CR, Hallegger M, Ule J, Marioni JC, Venkitaraman AR. Regulation of constitutive and alternative mRNA splicing across the human transcriptome by PRPF8 is determined by 5' splice site strength. Genome Biol. 2015;16:201. doi:10. 1186/s13059-015-0749-3.

43. Rivers C, Idris J, Scott H, Rogers M, Lee Y-B, Gaunt J, Phylactou L, Curk T, Campbell C, Ule J, et al. iCLIP identifies novel roles for SAFB1 in regulating RNA processing and neuronal function. BMC Biol. 2015;13:111. doi:10.1186/ s12915-015-0220-7.

44. Köster T, Meyer K, Weinholdt C, Smith LM, Lummer M, Speth C, Grosse I, Weigel D, Staiger D. Regulation of pri-miRNA processing by the hnRNP-like protein AtGRP7 in Arabidopsis. Nucleic Acids Res. 2014;42:9925-36. doi:10. 1093/nar/gku716.

45. Zhang J, Liu H, Sun J, Li B, Zhu Q, Chen S, Zhang H. Arabidopsis fatty acid desaturase FAD2 is required for salt tolerance during seed germination and early seedling growth. PLOS One. 2012;7:e30355. doi:10.1371/journal.pone.0030355.

46. Mittler R, Kim Y, Song L, Coutu J, Coutu A, Ciftci-Yilmaz S, Lee H, Stevenson B, Zhu J-K. Gain- and loss-of-function mutations in Zat10 enhance the tolerance of plants to abiotic stress. FEBS Lett. 2006;580:6537-42. doi:10. 1016/j.febslet.2006.11.002.

47. Nicaise V, Joe A, Jeong B, Korneli C, Boutrot F, Wested I, Staiger D, Alfano JR, Zipfel C. Pseudomonas HopU1 affects interaction of plant immune receptor mRNAs to the RNA-binding protein GRP7. EMBO J. 2013;32:701-12. doi:10. 1038/emboj.2013.15.

48. Fu ZQ, Guo M, Jeong BR, Tian F, Elthon TE, Cerny RL, Staiger D, Alfano JR. A type III effector ADP-ribosylates RNA-binding proteins and quells plant immunity. Nature. 2007;447:284-8. doi:10.1038/nature05737.

49. Perez-Santángelo S, Mancini E, Francey LJ, Schlaen RG, Chernomoretz A, Hogenesch JB, Yanovsky MJ. Role for LSM genes in the regulation of circadian rhythms. Proc Natl Acad Sci U S A. 2014;111:15166-71. doi:10. 1073/pnas.1409791111.

50. Nettling M, Treutler H, Grau J, Keilwagen J, Posch S, Grosse I. DiffLogo: a comparative visualization of sequence motifs. BMC Bioinf. 2015;16:387. doi: 10.1186/s12859-015-0767-x.

51. Alamancos GP, Pagès A, Trincado JL, Bellora N, Eyras E. Leveraging transcript quantification for fast computation of alternative splicing profiles. RNA. 2015:21:1521-31. doi:10.1261/rna.051557.115.

52. Drechsel G, Kahles A, Kesarwani AK, Stauffer E, Behr J, Drewe P, Rätsch G, Wachter A. Nonsense-mediated decay of alternative precursor mRNA splicing variants is a major determinant of the Arabidopsis steady state transcriptome. Plant Cell. 2013;25:3726-42. doi:10.1105/tpc.113.115485.

53. Streitner C, Simpson CG, Shaw P, Danisman S, Brown JWS, Staiger D. Small changes in ambient temperature affect alternative splicing in Arabidopsis thaliana. Plant Signal Behav. 2013;8:e24638. doi:10.4161/psb.24638.

54. Wachter A, Tunc-Ozdemir M, Grove BC, Green PJ, Shintani DK, Breaker RR. Riboswitch control of gene expression in plants by splicing and alternative $3^{\prime}$ end processing of mRNAs. Plant Cell. 2007;19:3437-50. doi:10.1105/tpc.107.053645.

55. Huppertz I, Attig J, D'Ambrogio A, Easton LE, Sibley CR, Sugimoto Y, Tajnik M, König J, Ule J. iCLIP: Protein-RNA interactions at nucleotide resolution. Methods. 2014;65:274-87. doi:10.1016/j.ymeth.2013.10.011.

56. Hauer C, Curk T, Anders S, Schwarzl T, Alleaume A-M, Sieber J, Hollerer I, Bhuvanagiri M, Huber W, Hentze MW, Kulozik AE. Improved binding site assignment by high-resolution mapping of RNA-protein interactions using iCLIP. Nat Commun. 2015;6:7921. doi:10.1038/ncomms8921.

57. Endo M, Shimizu H, Nohales MA, Araki T, Kay SA. Tissue-specific clocks in Arabidopsis show asymmetric coupling. Nature. 2014;514:419-22. doi:10.1038/ nature13919.

58. Inada N, Wildermuth MC. Novel tissue preparation method and cell-specific marker for laser microdissection of Arabidopsis mature leaf. Planta. 2005;221: 9-16. doi:10.1007/s00425-004-1427-y.

59. Marondedze C, Thomas L, Serrano NL, Lilley KS, Gehring C. The RNA-binding protein repertoire of Arabidopsis thaliana. Sci Rep. 2016;6:29766. doi:10. 1038/srep29766. 
60. Reichel M, Liao Y, Rettel M, Ragan C, Evers M, Alleaume A-M, Horos R, Hentze MW, Preiss T, Millar AA. In planta determination of the mRNAbinding proteome of Arabidopsis etiolated seedlings. Plant Cell. 2016;28 2435-52. doi:10.1105/tpc.16.00562.

61. Zhang Z, Boonen K, Ferrari P, Schoofs L, Janssens E, van Noort V, Rolland F, Geuten K. UV crosslinked mRNA-binding proteins captured from leaf mesophyll protoplasts. Plant Methods. 2016;12:42. doi:10.1186/s13007-016-0142-6.

62. Müller-McNicoll M, Botti $V$, de Jesus Domingues AM, Brandl H, Schwich OD, Steiner MC, Curk T, Poser I, Zarnack K, Neugebauer KM. SR proteins are NXF1 adaptors that link alternative RNA processing to mRNA export. Genes Dev. 2016;30:553-66. doi:10.1101/gad.276477.115.

63. Hauer C, Sieber J, Schwarzl T, Hollerer I, Curk T, Alleaume A-M, Hentze MW, Kulozik AE. Exon junction complexes show a distributional bias toward alternatively spliced mRNAs and against mRNAs coding for ribosomal proteins. Cell Rep. 2016;16:1588-603. doi:10.1016/j.celrep.2016.06.096.

64. Friedersdorf MB, Keene JD. Advancing the functional utility of PAR-CLIP by quantifying background binding to mRNAs and IncRNAs. Genome Biol. 2014;15:R2. doi:10.1186/gb-2014-15-1-r2.

65. Köster T, Marondedze C, Meyer K, Staiger D. RNA-binding proteins revisited - the emerging Arabidopsis mRNA interactome. Trends Plant Sci. 2017;22: 512-26. doi:10.1016/j.tplants.2017.03.009.

66. Uhl M, Houwaart T, Corrado G, Wright PR, Backofen R. Computational analysis of CLIP-seq data. Methods. 2017. doi:10.1016/j.ymeth.2017.02.006.

67. Zhang Y, Gu L, Hou Y, Wang L, Deng X, Hang R, Chen D, Zhang X, Zhang Y, Liu C, Cao X. Integrative genome-wide analysis reveals HLP1, a novel RNAbinding protein, regulates plant flowering by targeting alternative polyadenylation. Cell Res. 2015:25:864-76. doi:10.1038/cr.2015.77.

68. Uren PJ, Vo DT, de Araujo PR, Pötschke R, Burns SC, Bahrami-Samani E, Qiao M, de Sousa AR, Nakaya HI, Correa BR, et al. RNA-binding protein Musashi1 is a central regulator of adhesion pathways in glioblastoma. Mol Cell Biol. 2015;35:2965-78. doi:10.1128/mcb.00410-15.

69. Khan F, Daniëls MA, Folkers GE, Boelens R, Saqlan Naqvi SM, Ingen H. Structural basis of nucleic acid binding by Nicotiana tabacum glycine-rich RNA-binding protein: implications for its RNA chaperone function. Nucleic Acids Res. 2014;42:8705-18. doi:10.1093/nar/gku468.

70. Foley SW, Gosai SJ, Wang D, Selamoglu N, Sollitti AC, Köster T, Steffen A Lyons E, Daldal F, Garcia BA, et al. A global view of RNA-protein interactions identifies post-transcriptional regulators of root hair cell fate. Dev Cell. 2017; 41:204-20.e205. doi:10.1016/j.devcel.2017.03.018.

71. Kramer K, Sachsenberg T, Beckmann BM, Qamar S, Boon K-L, Hentze MW, Kohlbacher O, Urlaub H. Photo-cross-linking and high-resolution mass spectrometry for assignment of RNA-binding sites in RNA-binding proteins. Nat Methods. 2014;11:1064-70. doi:10.1038/nmeth.3092.

72. Sharma K, Hrle A, Kramer K, Sachsenberg T, Staals RHJ, Randau L, Marchfelder A, van der Oost J, Kohlbacher O, Conti E, Urlaub H. Analysis of protein-RNA interactions in CRISPR proteins and effector complexes by UVinduced cross-linking and mass spectrometry. Methods. 2015;89:138-48. doi: 10.1016/j.ymeth.2015.06.005.

73. Bieniawska Z, Espinoza C, Schlereth A, Sulpice R, Hincha DK, Hannah MA Disruption of the Arabidopsis circadian clock is responsible for extensive variation in the cold-responsive transcriptome. Plant Physiol. 2008;147:26379. doi:10.1104/pp.108.118059.

74. James $A B$, Syed NH, Bordage S, Marshall J, Nimmo GA, Jenkins Gl, Herzyk P, Brown JWS, Nimmo HG. Alternative splicing mediates responses of the Arabidopsis circadian clock to temperature changes. Plant Cell. 2012;24:961-81. doi:10.1105/tpc.111.093948.

75. Kershaw CJ, Costello JL, Talavera D, Rowe W, Castelli LM, Sims PFG, Grant CM, Ashe MP, Hubbard SJ, Pavitt GD. Integrated multi-omics analyses reveal the pleiotropic nature of the control of gene expression by Puf3p. Sci Rep. 2015:5:15518. doi:10.1038/srep15518.

76. Kaufmann K, Wellmer F, Muino JM, Ferrier T, Wuest SE, Kumar V, SerranoMislata A, Madueno F, Krajewski P, Meyerowitz EM, et al. Orchestration of floral initiation by APETALA1. Science. 2010;328:85-9. doi:10.1126/science.1185244.

77. Mateos J, Madrigal P, Tsuda K, Rawat V, Richter R, Romera-Branchat M, Fornara F, Schneeberger K, Krajewski P, Coupland G. Combinatorial activities of SHORT VEGETATIVE PHASE and FLOWERING LOCUS C define distinct modes of flowering regulation in Arabidopsis. Genome Biol. 2015;16:31. doi: 10.1186/s13059-015-0597-1.

78. Riley KJ, Steitz JA. The observer effect in genome-wide surveys of protein-RNA interactions. Mol Cell. 2013;49:601-4. doi:10.1016/j.molcel. 2013.01.030.
79. Michael TP, McClung CR. Enhancer trapping reveals widespread circadian clock transcriptional control in arabidopsis. Plant Physiol. 2003;132:629-39. doi:10.1104/pp.021006

80. Wuarin J, Falvey E, Lavery D, Talbot D, Schmidt E, Ossipow V, Fonjallaz P Schibler $U$. The role of the transcriptional activator protein DBP in circadian liver gene expression. J Cell Sci. 1992;16:123-7.

81. Staiger D. RNA-binding proteins and circadian rhythms in Arabidopsis thaliana. Philos Trans R Soc Lond B Biol Sci. 2001;356:1755-9. doi:10.1098/ rstb.2001.0964.

82. Schmal C, Reimann P, Staiger D. A circadian clock-regulated toggle switch explains AtGRP7 and AtGRP8 oscillations in Arabidopsis thaliana. PLoS Comput Biol. 2013;9:e1002986. doi:10.1371/journal.pcbi.1002986.

83. Rodor J, FitzPatrick DR, Eyras E, Cáceres JF. The RNA-binding landscape of RBM10 and its role in alternative splicing regulation in models of mouse early development. RNA Biol. 2017:14:45-57. doi:10.1080/15476286.2016.1247148.

84. Choudhary MK, Nomura Y, Shi H, Nakagami H, Somers DE. Circadian profiling of the Arabidopsis proteome using 2D-DIGE. Front Plant Sci. 2016; 7:1007. doi:10.3389/fpls.2016.01007.

85. Staiger D, Apel K. Circadian clock-regulated expression of an RNA-binding protein in Arabidopsis: characterisation of a minimal promoter element. Mol Gen Genet. 1999;261:811-9.

86. Heintzen C, Nater M, Apel K, Staiger D. AtGRP7, a nuclear RNA-binding protein as a component of a circadian-regulated negative feedback loop in Arabidopsis thaliana. Proc Natl Acad Sci U S A. 1997:94:8515-20.

87. Staiger D, Apel K, Trepp G. The Atger3 promoter confers circadian clockregulated transcription with peak expression at the beginning of the night. Plant Mol Biol Rep. 1999;40:873-82.

88. Chen B, Yun J, Kim MS, Mendell JT, Xie Y. PIPE-CLIP: a comprehensive online tool for CLIP-seq data analysis. Genome Biol. 2014;15:1-10. doi:10.1186/gb2014-15-1-r18.

89. Dobin A, Davis CA, Schlesinger F, Drenkow J, Zaleski C, Jha S, Batut P, Chaisson M, Gingeras TR. STAR: ultrafast universal RNA-seq aligner. Bioinformatics. 2013:29:15-21. doi:10.1093/bioinformatics/bts635.

90. Quinlan AR, Hall IM. BEDTools: a flexible suite of utilities for comparing genomic features. Bioinformatics. 2010;26:841-2. doi:10.1093/bioinformatics/btq033.

91. Patro R, Duggal G, Love Ml, Irizarry RA, Kingsford C. Salmon provides fast and bias-aware quantification of transcript expression. Nat Methods. 2017; 14:417-9. doi:10.1038/nmeth.4197.

92. Soneson C, Matthes KL, Nowicka M, Law CW, Robinson MD. Isoform prefiltering improves performance of count-based methods for analysis of differential transcript usage. Genome Biol. 2016;17:1-15. doi:10.1186/s13059-015-0862-3.

93. Pfaffl MW. A new mathematical model for relative quantification in real-time RT-PCR. Nucleic Acids Res. 2001;29:e45.

94. Lummer $M$, Humpert $F$, Wiedenlübbert $M$, Sauer $M$, Schüttpelz $M$, Staiger D. A new set of reversibly photoswitchable fluorescent proteins for use in transgenic plants. Mol Plant. 2013;6:1518-30. doi:10.1093/mp/sst040.

95. Heintzen C, Fischer R, Melzer S, Kappeler S, Apel K, Staiger D. Circadian oscillations of a transcript encoding a germin-like protein that is associated with cell walls in young leaves of the long-day plant Sinapis alba L. Plant Physiol. 1994;106:905-15.

96. Meyer K, Köster T, Nolte C, Weinholdt C, Lewinski M, Grosse I, Staiger D. Identification of AtGRP7 targets by iCLIP. 2017. https://www.ncbi.nlm.nih. gov/geo/query/acc.cgi?acc=GSE99427.

97. Meyer K, Köster T, Nolte C, Weinholdt C, Lewinski M, Grosse I, Staiger D. Identification of differentially expressed genes by RNA-seq in AtGRP7 overexpression and knockdown lines. 2017. https://www.ncbi.nlm.nih.gov/ geo/query/acc.cgi?acc=GSE99615.

98. Meyer K, Köster T, Nolte C, Weinholdt C, Lewinski M, Grosse I, Staiger D. Identification of AtGRP7 targets by RIP-seq. 2017. https://www.ncbi.nlm.nih. gov/geo/query/acc.cgi?acc=GSE99464.

99. Meyer K, Köster T, Nolte C, Weinholdt C, Lewinski M, Grosse I, Staiger D. Adaptation of iCLIP to plants determines the binding landscape of the clock-regulated RNA-binding protein AtGRP7. 2017. https://www.ncbi.nlm. nih.gov/geo/query/acc.cgi?acc=GSE99616. 